\title{
Late Quaternary fire regimes of Australasia
}

\author{
S.D. Mooney ${ }^{\mathrm{a}, *}$, S.P. Harrison ${ }^{\text {b }}$, P.J. Bartlein ${ }^{c}$, A.-L. Daniau ${ }^{\mathrm{d}}$, J. Stevenson ${ }^{\text {e }}$, K.C. Brownlie ${ }^{\text {f }}$, S. Buckman ${ }^{\mathrm{f}}$, \\ M. Cupper ${ }^{g}$, J. Luly ${ }^{\text {h }}$, M. Black ${ }^{\text {a }}$, E. Colhoun ${ }^{i}$, D. D’Costa ${ }^{j}$, J. Dodson ${ }^{k}$, S. Haberle ${ }^{\text {e }}$, G.S. Hope ${ }^{\mathrm{e}}$, P. Kershaw ${ }^{1}$, \\ C. Kenyon ${ }^{\mathrm{m}}$, M. McKenzie ${ }^{1}$, N. Williams ${ }^{\mathrm{n}}$
}

\author{
${ }^{a}$ School of Biological Earth and Environmental Sciences, University of New South Wales, Sydney, NSW 2052, Australia \\ ${ }^{\mathrm{b}}$ School of Biological Sciences, Macquarie University, North Ryde, NSW 2109, Australia \\ ${ }^{\mathrm{c}}$ Department of Geography, University of Oregon, Eugene, OR, USA \\ d School of Geographical Sciences, University of Bristol, Bristol, UK \\ e Department of Archaeology and Natural History, Australian National University, Canberra, ACT, Australia \\ ${ }^{\mathrm{f}}$ School of Earth and Environmental Sciences, University of Wollongong, NSW 2522, Australia \\ ${ }^{\mathrm{g}}$ School of Earth Sciences, The University of Melbourne, Victoria 3010, Australia \\ ${ }^{\mathrm{h}}$ School of Earth and Environmental Sciences, James Cook University, Townsville 4811, Australia \\ ${ }^{\mathrm{i}}$ School of Geosciences, University of Newcastle, Callaghan, NSW 2308, Australia \\ ${ }^{j}$ School of Environment, University of Auckland, Private Bag 92019, Auckland, New Zealand \\ ${ }^{\mathrm{k}}$ Australia Nuclear Science and Technology Organisation, Kirrawee, NSW 2232, Australia \\ ${ }^{1}$ School of Geography and Environmental Science, Monash University, Clayton, Victoria, Australia \\ ${ }^{\mathrm{m}}$ Melbourne School of Land and Environment, The University of Melbourne, Victoria 3010, Australia \\ ${ }^{\mathrm{n}}$ New South Wales Department of Environment, Climate Change and Water, Sydney, NSW 1232, Australia
}

\section{A R T I C L E I N F O}

Article history:

Received 19 July 2010

Received in revised form

13 October 2010

Accepted 15 October 2010

Available online $\mathrm{xxx}$

\begin{abstract}
A B S T R A C T
We have compiled 223 sedimentary charcoal records from Australasia in order to examine the temporal and spatial variability of fire regimes during the Late Quaternary. While some of these records cover more than a full glacial cycle, here we focus on the last 70,000 years when the number of individual records in the compilation allows more robust conclusions. On orbital time scales, fire in Australasia predominantly reflects climate, with colder periods characterized by less and warmer intervals by more biomass burning. The composite record for the region also shows considerable millennial-scale variability during the last glacial interval $(73.5-14.7 \mathrm{ka})$. Within the limits of the dating uncertainties of individual records, the variability shown by the composite charcoal record is more similar to the form, number and timing of Dansgaard-Oeschger cycles as observed in Greenland ice cores than to the variability expressed in the Antarctic ice-core record. The composite charcoal record suggests increased biomass burning in the Australasian region during Greenland Interstadials and reduced burning during Greenland Stadials. Millennial-scale variability is characteristic of the composite record of the subtropical high pressure belt during the past $21 \mathrm{ka}$, but the tropics show a somewhat simpler pattern of variability with major peaks in biomass burning around $15 \mathrm{ka}$ and $8 \mathrm{ka}$. There is no distinct change in fire regime corresponding to the arrival of humans in Australia at $50 \pm 10 \mathrm{ka}$ and no correlation between archaeological evidence of increased human activity during the past $40 \mathrm{ka}$ and the history of biomass burning. However, changes in biomass burning in the last 200 years may have been exacerbated or influenced by humans.
\end{abstract}

(c) 2010 Elsevier Ltd. All rights reserved.

\section{Introduction}

Australia includes some of the most fire-prone landscapes on Earth (Williams et al., 2001; Bradstock et al., 2002; Bond and Keeley, 2005; Russell-Smith et al., 2007). Fire has major impacts on the native flora and fauna, on landscape stability and on

\footnotetext{
* Corresponding author. Tel.: +61 293858063.

E-mail address: s.mooney@unsw.edu.au (S.D. Mooney).
}

biogeochemical cycling. Characteristics of the land cover and the release of gases and particulates $\left(\mathrm{CO}_{2}, \mathrm{CO}, \mathrm{CH}_{4}, \mathrm{~N}_{2} \mathrm{O}\right.$, BVOCs, black carbon) during bushfires affects air quality, atmospheric composition and hence radiation budgets, and thus changes in fire regimes through time could have important feedbacks to climate (Ramanathan and Carmichael, 2008; Bowman et al., 2009; Arneth et al., 2010).

Australian vegetation has developed a variety of responses and morphological and reproductive adaptations to fire, including the 
widespread use of resprouting (Purdie, 1977; Gill et al., 1981; Enright et al., 1998), suggesting that fire has played an important role over evolutionary timescales. Many species require regular fire in order to persist, and this is particularly evident in humid but intermittently drought-prone environments where eucalypts dominate the vegetation. Other taxa, including species typical of the more consistently humid east and northeastern coast, are fire sensitive (Gill et al., 1981; Bradstock et al., 2002). In the tropical communities of Australasia, fire often depends on drought and fires in the recent past have been linked to human activity and El Niño events (van der Werf et al., 2008a; Lynch et al., 2007). Fire is thus a fundamental issue in many Australasian landscapes and influences community composition, the location of boundaries between communities and vegetation dynamics through time.

There are persistent questions about the role of humans in the long-term history of fire and vegetation in the Australasian region. It has been argued that the frequent use of fire by Aboriginal people, to manipulate the availability of resources (Jones, 1969; Nicholson, 1981), resulted in vegetation change and other environmental impacts in the late Pleistocene (e.g. Singh et al., 1981; Flannery, 1994; Miller et al., 2005). Ideas about pre-historic fire continue to influence debates concerning natural resource management, with suggestions that Aboriginal-like fire management (i.e. frequent and low intensity fires) could prevent conflagrations in the modern setting (e.g. Select Committee on the Recent Australian Bushfires, 2003).

One of the earliest examinations of long-term changes in fire regimes and their impact on the development of Australian vegetation was provided by Gill et al. (1981). More recently, Kershaw et al. (2002) have summarised the long-term history of fire in Australia, although their discussion of the last 10,000 years focused solely on southeastern Australia and they used a qualitative assessment of ca. 60 sites. Nevertheless, Kershaw et al. (2002) indicated that there were differences in the timing of peak Holocene fires associated with different biomes across this region. The interval of least fire across all biomes was during the mid-Holocene (7000-5000 yr BP) and the maximum registration of fire occurred during the early European period. Lynch et al. (2007), which is the most recent review of Australian palaeofire regimes but only covers a small number of iconic records, also identifies the mid-Holocene as a time of low fire activity and argues that higher levels of biomass burning are associated with the onset or intensification of the El Niño-Southern Oscillation (ENSO) after ca. $4 \mathrm{ka}$. Lynch et al. (2007) conclude that the longer-term record of fire in Australia shows a gradual increase coincident with the purported long-term aridification of the continent (Hesse et al., 2004).

There have been several site specific or regional studies examining the interactions between vegetation and fire during the late Quaternary in Australia (e.g. Black et al., 2007). However, it is only recently, and largely through the efforts of the Global Palaeofire Working Group (GPWG: Power et al., 2008; Power et al., 2010), that sufficient data have become available at a continental scale to make it possible to apply robust statistical techniques to the analysis of past fire regimes. Power et al. (2008), based on an analysis of 355 charcoal records, showed that fire regimes globally reflected longterm climate changes. This compilation included only 48 records from "Australia", (defined as mainland Australia, New Zealand and Pacific Islands west of $180 \mathrm{oE}$, but not New Guinea which was included in southeast Asia). This preliminary analysis revealed distinctive patterns in fire regimes over the last $21 \mathrm{ka}$, with relatively little change during the late glacial and through the transition to the Holocene, and charcoal peaks around 15-16 ka, 11-10 ka and 4.5-2.5 ka (Power et al., 2008). Here, we present a more extensive synthesis of the charcoal data based on 223 sites from Australasia and analyse these data to determine how fire regimes have changed over centennial to multi-millennial timescales.

\subsection{Methods: source and treatment of charcoal records}

Our focus in this paper is on the Australasian region, which we define here to include tropical southeastern Asia, New Guinea, New Zealand and the islands of the western Pacific. Southeastern Asia and New Guinea were included to set the records from tropical Australia in a broader context. The inclusion of sites from the western Pacific (including New Zealand) is partly motivated by the need to address the potential role of changes in the ENSO on Australasian climates (McGlone et al., 1992; Shulmeister and Lees, 1995; Lynch et al., 2007) and partly because this region has a different settlement history from Australia (see e.g. Stevenson and Hope, 2005). We extracted 196 sedimentary charcoal records from this broadly defined Australasian region $\left(20^{\circ} \mathrm{N}-50^{\circ} \mathrm{S}\right.$ and $100^{\circ} \mathrm{E}$ to $177^{\circ} \mathrm{W}$ ) from Version 2 of the Global Charcoal Database (GCD-V2; Daniau et al., in preparation,) compiled by the Global Palaeofire Working Group (GPWG: http://gpwg.org/). These data were supplemented by an additional 27 sites, chosen to increase the number of long records and to improve the spatial coverage (GCD-V2.5).

This GPWG database contains sedimentary charcoal records from both marine and terrestrial sites. It includes descriptive data (metadata) about both the sites and the methods used, and detailed information on site chronology (including information on the number of radiometric dates and, for records extending back beyond the limits of radiocarbon dating, details of correlative tiepoints used to erect the chronology). All radiocarbon dates have been calibrated and the age model for each site is expressed in calendar years. Where multiple records (e.g. macro and microcharcoal records) are available at the same site, all are included in the database.

Since charcoal records are obtained using many different techniques and expressed using a large range of metrics, the data were standardized to facilitate comparisons between sites and through time (see Power et al., 2008 for a full description). The protocol involved three steps. First, non-influx data (e.g. concentration expressed as particles $/ \mathrm{cm}^{3}$; charcoal-to-pollen ratios) were transformed to influx values (i.e. particles $/ \mathrm{cm}^{2} / \mathrm{yr}$ ), or quantities proportional to influx, by dividing the values by sample deposition times. Second, a Box-Cox transformation was used to homogenize the inter-site variance by transforming individual charcoal records toward normality. Finally, the data were rescaled using a common base period $(0.2-21 \mathrm{ka})$ to yield $\mathrm{z}$-scores, so that all sites have a common mean and variance. Previous experimentation has demonstrated that the choice of the base period does not affect the results significantly (Marlon et al., 2008; Power et al., 2010) and standardization does not alter the overall pattern of variability or "signal" in a record. For mapping purposes, the z-scores were divided into five approximately equal groups: $z$-score $>+0.8$ (strong positive anomalies compared to the long-term average over the base period), $z$-score between +0.4 and +0.8 (positive anomalies), z-score between +0.4 and -0.4 (weak positive or negative anomalies), $z$-score between -0.4 and -0.8 (negative anomalies), and $\mathrm{z}$-score $<-0.8$ (strong negative anomalies).

In order to summarise the broadest-scale history of changing fire regimes through time, we constructed composite charcoal records for Australasia as a whole and for various sub-regions. Composite curves were then obtained by fitting a locally weighted regression (or "lowess" curve) to the pooled transformed and rescaled data (see e.g. Marlon et al., 2008), using a fixed window width and a tricube weight function with one "robustness iteration". We used window half-widths of $100,200,400$, or 2000 years, to emphasize different scales of temporal variability from centennial variability in the past 2000 years through to multi-millennial variability in the longer records. The windows were selected to avoid both over-smoothing, as would result from selecting a large 
window width, and under-smoothing, leading to a composite curve that was susceptible to the influence of individual data points. These choices do not affect the results and conclusions.

Confidence intervals for each composite curve were generated by a bootstrap re-sampling (with replacement) of individual sites over 1000 replications. This approach differs from the usual bootstrap method in which individual observations are sampled with replacement, and emphasizes the uncertainty in regression curves that arises from the inclusion or exclusion of the whole record from individual sites. Bootstrap confidence limits for each target point were taken as the 2.5th and 97.5th percentiles of the 1000 fitted values for that target point. Our approach is therefore conservative because it permits the identification of "signals" that may arise solely from the influence of a particular site: when the bootstrap confidence intervals are wide, this indicates greater uncertainty in the composite curve and greater sensitivity of that curve to the addition/subtraction of an individual record. Minor fluctuations in the composite curve during times when the bootstrap confidence intervals are wide are most probably meaningless. Most of the time, the composite curve lies in the middle of the bootstrap confidence intervals; when one confidence limit deviates from the composite curve more than the other confidence limit, this too indicates that some individual records depart markedly from the typical pattern of the time. Again, the interpretation of minor fluctuations in the composite curve under these circumstances is not likely to be meaningful. We also provide a measure of the number of observations contributing to the composite curve (at each of the target points that define the curve) as an additional measure of the confidence to be placed in the reconstructions. Since this takes into account the number of points at each site that fall within the window width for a particular target point, these curves are not a simple measure of the number of sites.

\section{Results}

\subsection{Characteristics of the data set}

The geographic coverage of sites in the dataset is uneven (Fig. 1), with few sites from central and western Australia, but with eastern and especially southeastern, Australia well represented. While there are some sites from tropical mainland Australia, most of the tropical records are from Papua New Guinea, with a small number of additional sites scattered throughout southeastern Asia as well as several tropical Pacific Islands (Fig. 1a). The coverage of records from New Zealand is reasonable, although it does not approach the known number of records for the island. The paucity of data from northern, western and central Australia is partially a reflection of absence of suitable sites for the preservation of sedimentary charcoal records (Pickett et al., 2004) and partially a function of the comparative difficulty of working in these regions. Nevertheless, the data do provide a reasonably comprehensive coverage of the range of climates and vegetation types found in Australasia (Fig. 1a-e).

The temporal coverage of the data set is also uneven (Table 1). There are 18 records that extend back beyond $70 \mathrm{ka}$ (Fig. 1c). These sites are not spatially clustered, but rather occur from just north of the equator to the South Island of New Zealand, and therefore experienced very different climate and fire regimes from one another. There are 19 sites that provide a record from the beginning of Marine Isotope Stage (MIS) 3 (here defined as $59.4 \mathrm{ka}$, following Sanchez Goñi and Harrison, 2010) and 40 sites that are recording by the end of MIS 3 (here defined as $27.8 \mathrm{ka}$, following Sanchez Goñi and Harrison, 2010). Over half of these records are sampled at an average resolution of more than 1 sample per ka (Table 1 ). The number of records and their spatial distribution during MIS 3 makes Australasia one of the best-documented regions of the world for this period (see Daniau et al., 2010). The number of charcoal records increases steadily during MIS $2(27.8-14.7 \mathrm{ka})$, such that by the end of MIS 2 there are approximately 70 sites and by 10 ka there are about 110 sites recording fire. Sampling resolution for sites covering the Holocene varies from ca 1 sample per ka to 1 sample per decade: the majority of the short records (i.e. those covering the last 1-2 ka) have been sampled at decadal resolution, while the majority of the late Holocene records have centennial resolution.

The charcoal records from Australasia were obtained using several different techniques (Table 1). The quantification of charcoal fragments on pollen slides dominates the original records ( $80.4 \%$ of the sites where method was explicitly recorded). However, 44 of the records ( $19.6 \%$ of the sites where method was explicitly recorded) are macroscopic charcoal records obtained by wet sieving. Macroscopic, sieved, charcoal records are more likely to provide a record of local fires and provide more continuous temporal sequences than charcoal records obtained through other means. Nevertheless, although differences between the macro- and micro-charcoal records at individual sites have been used to infer the spatial scale of fire events (Power et al., 2010), both microscopic and macroscopic records produce comparable results in terms of broadscale regional histories of fire (Tinner et al., 2006; Conedera et al., 2009).

\subsection{Temporal trends in biomass burning}

There are relatively few sites with a record prior to MIS 4 and the composite curve for the interval prior to $70 \mathrm{ka}$ is too noisy to interpret. We have therefore chosen to focus our analyses on the interval after $70 \mathrm{ka}$. The composite record from Australasia (Fig. 2, purple curve) shows a steady increase in biomass burning during the later part of MIS 4 (73.5-59.4 ka). Biomass burning remains generally high through MIS 3, but decreases at the beginning of MIS 2. Biomass burning levels are generally high through the Holocene. This pattern, of higher levels of biomass burning during MIS 3 than MIS 4, the reduction of biomass burning at the beginning of MIS 2 , and the return to higher levels of biomass burning during the Holocene, is consistent with the global pattern of less fire during cold stadial or glacial stages, and increased fire during warmer interstadials and interglacials (Power et al., 2008; Daniau et al., 2010). Although there are marked changes in fire activity during MIS 3 (see below), there is no fundamental shift in the composite charcoal record that could be associated with the colonization of Australia by Aboriginal people ( $50 \pm 10 \mathrm{ka}$ : Bird et al., 2004).

Superimposed on these general trends, the composite record shows considerable millennial-scale variability in biomass burning (Fig. 2, black curve). This is most marked in MIS 3, but can also be seen during the later part of MIS 4, with multiple peaks in biomass burning in the 400-year smoothed record between 70 and $20 \mathrm{ka}$ (Fig. 2, black curve). Some of these charcoal peaks appear to occur around the time of the Dansgaard-Oeschger (D-O) warming events as described in Greenland ice-core records (Fig. 3). Other peaks in the charcoal record are not apparently coincident with $\mathrm{D}-\mathrm{O}$ warming events, but this may be a function of the considerable uncertainty associated with the chronologies of individual charcoal records that are beyond the limit of radiocarbon dating. Several of the records contributing to the composite curve have been tuned to match assumed limits of the transition between the last interglacial and the glacial (MIS 5/4 boundary) and the MIS 3/2 boundary on the basis of changes in pollen stratigraphy, and this procedure could certainly add uncertainties of several thousand years to the assumed age model. In terms of the number and shape of the peaks, and in terms of the timing during the post-30 ka interval (when radiocarbon dating is likely to be more reliable), the 

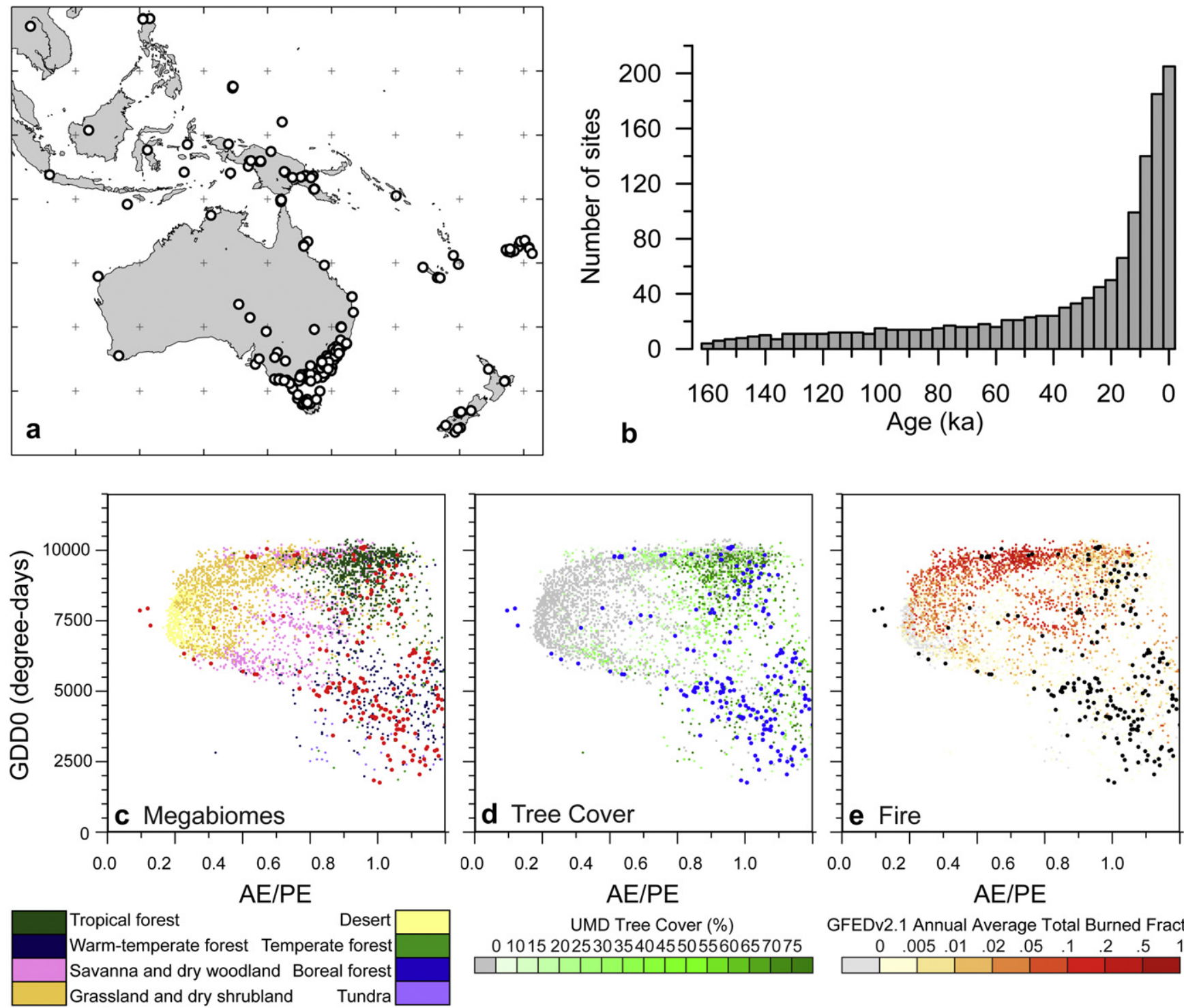

$A E / P E$

UMD Tree Cover (\%) 01015202530354045505560657075
GFEDv2.1 Annual Average Total Burned Fraction

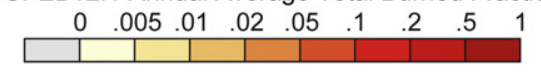



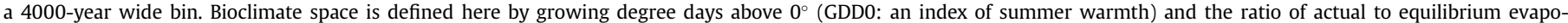

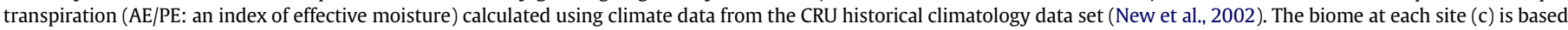

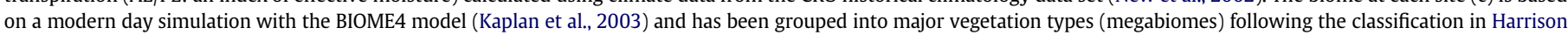

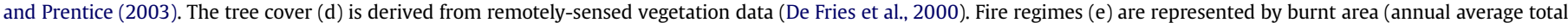
burnt fraction) from the GFED v2.1 data set (van der Werf et al., 2006).

form of the composite charcoal curve is more similar to the pattern of millennial-scale variability shown in the NGRIP record than to the pattern shown by the EPICA deuterium record (Fig. 3), which shows a smaller number of lower amplitude "Antarctic Isotope Maxima" over the same interval (EPICA Community Members, 2006). Furthermore, this pattern is consistent with a global analysis of charcoal records that span the interval from 80 ka to $10 \mathrm{ka}$ (Daniau et al., 2010) that show a consistent (across the D-O events) increase in biomass burning accompanying $\mathrm{D}-\mathrm{O}$ warming events.

There are relatively low levels of biomass burning between ca 24 to $18 \mathrm{ka}$ (i.e. around the time of the Last Glacial Maximum, LGM), but fire increases thereafter. The post-glacial increase of biomass burning (Fig. 4a) is expressed differently in the Intertropical Convergence Zone belt (ITCZ, $20^{\circ} \mathrm{N}$ to $20^{\circ} \mathrm{S}, 100^{\circ} \mathrm{E}$ to $177^{\circ} \mathrm{W}$ ) and the sub-tropical high pressure belt $\left(\mathrm{STH}, 25-45^{\circ} \mathrm{S}, 100^{\circ} \mathrm{E}\right.$ to $177^{\circ}$ $\mathrm{W})$. In the tropics (Fig. 4b), biomass burning increases gradually after the LGM through to the early Holocene, but with two broad peaks of increased fire centered around $15 \mathrm{ka}$ and $8 \mathrm{ka}$. There is a sharp decrease in biomass burning culminating at $6.5 \mathrm{ka}$. There is a rapid recovery after this and the rest of the Holocene appears relatively complacent until the last few hundred years. The records from the STH belt (Fig. 4c) show a different pattern, with no marked trends during the deglacial period, but there is a tendency towards increased biomass burning during the first part of the Holocene culminating around $6 \mathrm{ka}$ and a decline in fire during the late Holocene. The STH record also shows centennial- to millennialscale variability throughout the last $21 \mathrm{ka}$. Again, the most pronounced changes in STH fire regimes occur during the last few hundred years.

The composite Australasian record of biomass burning over the past two millennia (Fig. 5a) is remarkably flat except for the pronounced increase in fire in the past 200 years. Marlon et al. (2008) showed that global biomass burning gradually declined over most of the last two millennia in response to long-term 
Sites with charcoal records from the Australasian region.

\begin{tabular}{|c|c|c|c|c|c|c|c|c|}
\hline Site Name & Latitude $\left(^{\circ}\right)$ & Longitude $\left(^{\circ}\right)$ & Elevation (m) & Site type & Charcoal methods & $\begin{array}{l}\text { Record length } \\
\text { (age ka) }\end{array}$ & $\begin{array}{l}\text { Resolution } \\
\text { (samples/ka) }\end{array}$ & References \\
\hline Aguai Ramata & -6.5639 & 145.211 & 1950 & lacustrine & macro, sieved & 15.84 & 32.88 & Haberle, 2007 \\
\hline Aire Crossing & -38.6467 & 143.4797 & 180 & terrestrial & micro, pollen slide & 10.33 & 3.97 & McKenzie and Kershaw, 2004 \\
\hline Ajax Hill & -46.4192 & 169.2922 & 680 & mire & macro, sieved & 14.21 & 4.01 & McGlone, 2009 \\
\hline Ajkwa 1 & -4.867 & 136.968 & 1.3 & coastal & micro, pollen slide & 6.16 & 4.38 & Ellison, 2005 \\
\hline Ajkwa 2 & -4.867 & 136.968 & 1.3 & coastal & micro, pollen slide & 10.43 & 5.85 & Ellison, 2005 \\
\hline Ajkwa 3 & -4.867 & 136.968 & 1.3 & coastal & micro, pollen slide & 2.96 & 13.83 & Ellison, 2005 \\
\hline Ajkwa 4 & -4.867 & 136.968 & 1.3 & coastal & micro, pollen slide & 5.55 & 4.86 & Ellison, 2005 \\
\hline Ajkwa 5 & -4.867 & 136.968 & 1.3 & coastal & micro, pollen slide & 5.55 & 7.38 & Ellison, 2005 \\
\hline Allom Lake & -25.2333 & 153.1667 & 100 & lacustrine & macro, sieved & 57.00 & 2.14 & Donders et al., 2006 \\
\hline Anggi Lake & -1.403 & 133.902 & 1945 & lacustrine & micro, pollen slide & 26.08 & 0.35 & Hope, 2007a \\
\hline Anumon Swamp & -20.163 & 169.828 & 45 & mire & micro, pollen slide & 1.87 & 4.27 & Hope, 1996b \\
\hline Argan Swamp & -10.05 & 142.06 & 3 & coastal & macro, sieved & 5.52 & 23.74 & Rowe, 2006a \\
\hline Aru & -5.917 & 134.2 & 1 & terrestrial & micro, pollen slide & 6.89 & 2.61 & Hope and Aplin, 2005 \\
\hline Badu 15 & -10.06 & 142.09 & 20 & terrestrial & macro, sieved & 4.19 & 7.63 & Rowe, 2006a, b, 2007 \\
\hline Banda Sea Core SHI-9014 & -5.76667 & 126.9667 & -3163 & marine & micro, pollen slide & 173.31 & 0.54 & van der Kaars et al., 2000 \\
\hline Bar20 & -10.1 & 142.12 & 18 & coastal & macro, sieved & 2.83 & 25.42 & Rowe, 2006a, 2007 \\
\hline Bega Swamp & -36.5167 & 149.5 & 1080 & mire & micro, pollen slide & 12.25 & 39.92 & Polach and Singh, 1980 \\
\hline Black Swamp & -30.0206 & 151.4828 & 1450 & mire & micro, pollen slide & 10.62 & 6.21 & Dodson et al., 1986 \\
\hline Blue Lake Kosciuzko & -36.141 & 148.437 & 1950 & lacustrine & macro, sieved & 8.18 & 60.43 & Raine, 1974 \\
\hline Blundells Flat & -35.19 & 148.49 & 762 & mire & macro, sieved & 2.69 & 90.70 & Hope et al., 2006b \\
\hline Bobundara Swamp & -36.25 & 150.06 & 75 & coastal & micro, pollen slide & 8.01 & 9.99 & Hope et al., 2006a \\
\hline Boggy Swamp & -29.9611 & 151.4986 & 1160 & mire & micro, pollen slide & 12.49 & 6.73 & Dodson et al., 1986 \\
\hline Boigu Gawat Core 1 & -10.1 & 142.14 & 10 & coastal & macro, sieved & 4.57 & 10.94 & Rowe, 2006a, 2007 \\
\hline Boigu Gawat Core 2 & -10.1 & 142.14 & 10 & coastal & macro, sieved & 13.82 & 3.62 & Rowe, 2007 \\
\hline Bolin Billabong combined core & -37.7667 & 145.0667 & 50 & fluvial & micro, pollen slide & 0.87 & 46.91 & Leahy et al., 2005 \\
\hline Bonatoa & -18.07 & 178.53 & 4 & mire & micro, pollen slide & 5.41 & 5.18 & Hope, 1996a; Hope et al., 1999 \\
\hline Bondi Lake Centre Core & -36.8061 & 149.9378 & 22 & coastal & micro, pollen slide & 1.16 & 34.40 & Dodson et al., 1993 \\
\hline Bondi Lake South Core & -36.8061 & 149.9378 & 22 & coastal & micro, pollen slide & 2.12 & 13.19 & Dodson et al., 1993 \\
\hline Boulder Flat & -37.45 & 148.92 & 25 & terrestrial & micro, pollen slide & 27.22 & 1.25 & Kenyon, 1989 \\
\hline Breadalbane & -34.8 & 149.5167 & 694 & lacustrine & micro, pollen slide & 6.50 & 2.62 & Dodson, 1986 \\
\hline Bridgewater Lake Core B & -38.3 & 141.3833 & 20 & lacustrine & micro, pollen slide & 4.86 & 7.62 & Head, 1988 \\
\hline Brooks Ridge Fen & -36.1583 & 148.5944 & 1450 & mire & micro, pollen slide & 1.65 & 8.48 & Mooney et al., 1997 \\
\hline Burraga Swamp & -32.0281 & 151.4334 & 1462 & mire & micro, pollen slide & 1.87 & 17.16 & Dodson et al., 1994c \\
\hline Burralow Creek Swamp & -33.5333 & 150.6333 & 320 & mire & micro, pollen slide & 1.05 & 12.34 & Chalson, 1991 \\
\hline Butchers Swamp & -29.9722 & 151.4522 & 1230 & mire & micro, pollen slide & 14.21 & 2.25 & Dodson et al., 1986 \\
\hline Buxton & -37.4483 & 145.6919 & 235 & mire & micro, pollen slide & 7.20 & 5.83 & McKenzie, 2002 \\
\hline Byenup Lagoon Site 1 & -34.4667 & 116.7333 & 175 & lacustrine & micro, pollen slide & 4.90 & 3.67 & Dodson and Lu., 2000 \\
\hline Byenup Lagoon Site 2 & -34.4667 & 116.7167 & 175 & lacustrine & micro, pollen slide & 13.20 & 1.36 & Dodson and Lu., 2000 \\
\hline Caledonia Fen & -37.3333 & 146.7333 & 1280 & mire & micro, pollen slide & 140.72 & 1.21 & Kershaw et al., 2007 \\
\hline Cameron's Lagoon & -41.967 & 146.683 & 1045 & mire & micro, pollen slide & 7.83 & 2.68 & Thomas and Hope, 1994 \\
\hline Chapple Vale Swamp & -38.6261 & 143.323 & 30 & mire & micro, pollen slide & 7.16 & 9.64 & McKenzie and Kershaw, 1997 \\
\hline Clarks Junction & -45.7181 & 170.1139 & 560 & lacustrine & macro, sieved & 16.80 & 2.20 & McGlone, 2001 \\
\hline Club Lake & -36.4 & 148.3166 & 1980 & lacustrine & micro, pollen slide & 0.84 & 64.06 & Dodson et al., 1994a \\
\hline Cobrico Swamp & -38.3 & 143.0333 & 140 & mire & micro, pollen slide & 0.64 & 15.71 & Dodson et al., 1994b \\
\hline Core Fr10/95-GC-17 & -22.0458 & 113.5018 & -1093 & marine & micro, pollen slide & 101.64 & 0.96 & Van der Kaars and De Deckker, 2002 \\
\hline Cotter Source Bog margin & -35.9667 & 148.8167 & 1755 & mire & micro, pollen slide & 10.68 & 1.68 & Jones, 1990 \\
\hline Cotter Source Bog center & -35.9667 & 148.8167 & 1755 & mire & micro, pollen slide & 6.93 & 2.31 & Jones, 1990; Hope and Clark, 2008 \\
\hline Cuddie Springs & -30.3795 & 147.3117 & 127 & lacustrine & micro, pollen slide & 57.45 & 0.54 & Field et al., 2002 \\
\hline Dalhousie Springs & -26.4167 & 135.52 & 150 & spring & micro, pollen slide & 1.96 & 9.71 & Boyd, 1990 \\
\hline Den Plain 3 & -41.3 & 146.2 & 350 & lacustrine & micro, pollen slide & 2.38 & 5.04 & Moss et al., 2007 \\
\hline Doge Doge & -17.9066 & 177.2776 & 8 & fluvial & micro, pollen slide & 8.58 & 1.86 & Hope et al., 2009 \\
\hline Dove Lake & -41.66 & 145.96 & 934 & lacustrine & micro, pollen slide & 13.50 & 5.70 & Dyson, 1995 \\
\hline Dublin Bog & -41.727 & 146.233 & 710 & mire & macro, sieved & 15.90 & 5.79 & Colhoun et al., 1991 \\
\hline
\end{tabular}




\begin{tabular}{|c|c|c|c|c|c|c|c|c|}
\hline Site Name & Latitude $\left(^{\circ}\right)$ & Longitude $\left(^{\circ}\right)$ & Elevation (m) & Site type & Charcoal methods & $\begin{array}{l}\text { Record length } \\
\text { (age ka) }\end{array}$ & $\begin{array}{l}\text { Resolution } \\
\text { (samples/ka) }\end{array}$ & References \\
\hline Egg Lagoon & -39.65 & 143.95 & 20 & mire & micro, pollen slide & 149.38 & 0.74 & D'Costa et al., 1993 \\
\hline Evoran Pond & -18.7613 & 169.0118 & 194 & lacustrine & micro, pollen slide & 2.56 & 10.92 & G. Hope, unpublished data \\
\hline Eweburn Bog & -45.32 & 167.8089 & 320 & mire & micro, pollen slide & 10.61 & 5.75 & Ogden et al., 1998 \\
\hline Fred South Swamp & -38.1368 & 141.7835 & 27 & mire & micro, pollen slide & 18.03 & 1.16 & Builth et al., 2008 \\
\hline Gallahers Swamp & -34.29 & 150.43 & 535 & mire & micro, pollen slide & 22.21 & 0.90 & Hope, 2005a \\
\hline Galway Tarn & -43.4083 & 169.8733 & 130 & lacustrine & macro, sieved & 50.86 & 2.36 & M. Vandergoes, unpublished data \\
\hline Ginini Flats & -35.31 & 148.46 & 1590 & mire & micro, pollen slide & 3.45 & 4.35 & Hope et al., 2005 \\
\hline Glendhu Bog & -45.8381 & 169.7264 & 600 & mire & macro, sieved & 12.00 & 5.17 & McGlone and Wilmshurst, 1999 \\
\hline Goochs Swamp & -33.45 & 150.26 & 960 & mire & macro, sieved & 14.06 & 5.12 & Black and Mooney, 2006 \\
\hline Greens Bush & -38.4333 & 144.9333 & 160 & coastal & micro, pollen slide & 5.77 & 4.85 & Jenkins, 1992 \\
\hline $\begin{array}{l}\text { Grey Pole Swamp } \\
\text { Broughton Island }\end{array}$ & -32.6149 & 152.3173 & 9 & mire & macro, sieved & 5.38 & 46.44 & Horn, 2005 \\
\hline Griffith Swamp & -33.2833 & 151 & 20 & mire & macro, sieved & 6.43 & 42.33 & Mooney et al., 2007 \\
\hline Haeapugua & -5.8333 & 142.7833 & 1650 & marsh & micro, pollen slide & 25.50 & 3.22 & Haberle and Ledru, 2001 \\
\hline Henty Bridge & -41.9922 & 145.4736 & 115 & spring & micro, pollen slide & 29.42 & 0.61 & Colhoun, 1985 \\
\hline Hogan's Billabong & -36.025 & 146.7153 & 140 & lacustrine & micro, pollen slide & 1.92 & 18.23 & Reid et al., 2007 \\
\hline Hogayaku & -3.983 & 137.383 & 3580 & lacustrine & micro, pollen slide & 5.37 & 10.43 & Hope, 2007b \\
\hline Hopwoods Lagoon & -33.217 & 150.9933 & 38 & lacustrine & macro, sieved & 0.66 & 60.94 & Smeulders, 1999 \\
\hline Howes Waterhole Swamp & -33.0167 & 150.6667 & 280 & mire & macro, sieved & 2.37 & 75.95 & Mason, 2004 \\
\hline Ijomba & -4.0333 & 137.2167 & 3630 & mire & micro, pollen slide & 17.91 & 2.46 & Haberle and Ledru, 2001 \\
\hline Ingar Swamp & -33.7699 & 150.4563 & 584 & mire & micro, pollen slide & 8.01 & 2.62 & Chalson, 1991 \\
\hline Jibbon Lagoon & -34.0833 & 151.15 & 65 & mire & macro, sieved & 1.58 & 48.75 & Mooney et al., 2001 \\
\hline Kaipo & -38.4 & 177.1 & 555 & mire & micro, pollen slide & 17.51 & 7.77 & $\begin{array}{l}\text { Newnham and Lowe, 2000; } \\
\text { Hajdas et al., } 2006\end{array}$ \\
\hline Katoomba Swamp & -33.7173 & 150.3189 & 950 & mire & micro, pollen slide & 7.16 & 3.63 & Chalson, 1991 \\
\hline Kelela Swamp & -4.0207 & 138.9125 & 1650 & mire & micro, pollen slide & 11.67 & 3.17 & Haberle et al., 1991 \\
\hline Kettlehole Bog & -43.0546 & 171.7862 & 600 & mire & micro, pollen slide & 17.45 & 5.16 & McGlone et al., 2004 \\
\hline Killalea Lagoon & -34.6003 & 150.8678 & 22 & coastal & micro, pollen slide & 1.93 & 12.44 & Dodson et al., 1993 \\
\hline Kings Tableland Swamp & -33.7333 & 150.4833 & 780 & mire & micro, pollen slide & 18.39 & 1.36 & Chalson, 1991 \\
\hline $\begin{array}{l}\text { Kings Tableland Swamp } \\
\text { (short core) }\end{array}$ & -33.7333 & 150.4833 & 780 & mire & macro, sieved & 1.06 & 94.54 & Chalson, 1991; Black, 2001 \\
\hline Kings Waterhole & -33.0167 & 150.6667 & 280 & mire & macro, sieved & 6.30 & 17.15 & Black, 2001 \\
\hline Kohuora & -36.57 & 174.52 & 73 & lacustrine & micro, pollen slide & 32.43 & 4.87 & Newnham et al., 2007a \\
\hline Kosipe A & -8.4667 & 147.2 & 1960 & marsh & micro, pollen slide & 33.36 & 0.87 & Hope, 2009 \\
\hline Kosipe C & -8.4667 & 147.2 & 1960 & marsh & micro, pollen slide & 55.00 & 0.80 & Hope, 2009 \\
\hline Koumac & -20.65 & 164.283 & 2 & coastal & micro, pollen slide & 6.38 & 0.94 & Hope et al., 1999 \\
\hline Kurnell Fen & -34.01 & 151.1 & 15 & coastal & micro, pollen slide & 9.19 & 3.59 & Martin, 1994 \\
\hline Kurnell Swamp & -34.0333 & 151.2167 & 2 & mire & micro, pollen slide & 2.00 & 5.50 & Martin, 1994 \\
\hline Lac Suprin & -22.18 & 166.59 & 230 & lacustrine & micro, pollen slide & 33.17 & 1.42 & Hope and Pask, 1998 \\
\hline Lake Baraba Thirlmere Lakes & -34.2342 & 150.5397 & 305 & lacustrine & macro, sieved & 54.60 & 1.70 & Black et al., 2006 \\
\hline Lake Condah & -38.0667 & 141.8333 & 60 & lacustrine & micro, pollen slide & 11.18 & 2.95 & Builth et al., 2008 \\
\hline Lake Coomboo & -25.2195 & 153.1959 & 90 & lacustrine & micro, pollen slide & 202.31 & 0.31 & Longmore, 1997 \\
\hline Lake Couridjah Thirlmere Lakes & -34.2322 & 150.542 & 310 & lacustrine & macro, sieved & 15.42 & 1.95 & Clark, 1997 \\
\hline Lake Curlip & -37.8333 & 148.565 & 2 & lacustrine & micro, pollen slide & 0.32 & 99.96 & Ladd, 1978 \\
\hline Lake Euramoo & -17.1599 & 145.6286 & 718 & lacustrine & macro, sieved & 23.48 & 32.71 & Haberle, 2005 \\
\hline Lake Eyre (Core LE 82-2) & -28.5 & 137.25 & -15 & lacustrine & micro, pollen slide & 39.18 & 1.51 & Gillespie et al., 1991; Luly, 2001 \\
\hline Lake Flannigan King Island & -39.6 & 143.95 & 40 & mire & micro, pollen slide & 4.05 & 4.69 & D'Costa, 1997 \\
\hline Lake Frome & -30.68 & 139.78 & 40 & lacustrine & micro, pollen slide & 22.68 & 5.07 & $\begin{array}{l}\text { Singh and Luly, 1991; Luly and Jacobsen, } \\
\text { 2000; Luly, } 2001\end{array}$ \\
\hline Lake George & -35.0656 & 149.4181 & 673 & lacustrine & micro, pollen slide & 116.71 & 0.58 & $\begin{array}{l}\text { Singh et al., } 1981 \text {; Singh } \\
\text { and Geissler, } 1985\end{array}$ \\
\hline Lake Habbema & -4.1167 & 138.7 & 3120 & lacustrine & micro, pollen slide & 11.59 & 3.80 & Haberle et al., 2001 \\
\hline Lake Hordern & -38.7833 & 143.4667 & 3 & lacustrine & micro, pollen slide & 4.80 & 4.59 & Head and Stuart, 1980 \\
\hline Lake Hordorli & -2.533 & 140.55 & 680 & mire & micro, pollen slide & 63.45 & 0.95 & Hope and Tulip, 1994 \\
\hline Lake Johnston & -41.8666 & 145.55 & 900 & lacustrine & micro, pollen slide & 11.72 & 4.52 & Dodson et al., 1998; Anker et al., 2001 \\
\hline Lake Majo & -1.46667 & 127.4833 & 140 & lacustrine & micro, pollen slide & 5.80 & 4.14 & Haberle and Ledru, 2001 \\
\hline Lake Mountain & -37.469 & 145.875 & 1450 & mire & micro, pollen slide & 8.66 & 2.42 & McKenzie, 1997 \\
\hline
\end{tabular}


145.6

Lake Tyrrell1

$-35.31$

142.78

516
93
42

lacustine

micro, pollen slide
micro, pollen slide

130.24
33.30

17.46

lacustrine

Lake Wangoom LW87 core

Laravita

Lashmars Lagoon

Laukutu Swamp

Loch Sport Swamp

Lombok Ridge Core G6-4

Long Swamp

Lynchs Crater
Lynchs Crater (holocene core)

Mago Island

Main Lake Tower Hill

Maluyo Swamp

McKenzie Road Bog

MD97-2140

Mela Swamp

Micalong Swamp

Middle Patriarch Swamp

Mill Creek

Mountain Lagoon

Muellers Rock

Mulloon

Nadrau

Native Companion Lagoon

Navatu

Nekkeng

Newall Creek

Newnes Swamp

Ngardmau

Ngerchau

Ngerdok 2

Ngerkell

Nong Pa Kho

Noreikora Swamp

North Torbreck

Northwest Crater Tower Hill

Notts Swamp

Nursery Swamp

Oaks Creek

820

Okarito Pakihi

Olbed 1

Paoay Lake LP3

Paoay Lake LP4

Pemerak Swam

Penrith Lakes

Pine Camp

Plum Swamp

Poets Hill

Poley Creek
Powelltown
142.78

42

$\begin{array}{ll}-38.35 & 142.6 \\ -8.3909 & 147.352\end{array}$

$-35.8$

$-9.4794$

$-37.9666$

$-38.0833$

$-38.0833$

$-17.3667$

$-17.44$

$-38.3167$

18.18

$-38.4333$

2.0667
-9.4794

$-35.3333$

$-39.998$

$-33.404$

$-33.5$

-35.39
-35.4417

-35.4417
-17.75

$-27.6754$

$-18.07$

7.45
-8.4725

-8.4725
-42.07

$-33.3825$

7.608

7.52

7.605292

17.01

$-6.3333$

$-37.4814$

$-33.3098$

$-35.41$

$-37.5856$

$-16.6333$

$-43.2417$

7.5

18.2
18.12

0.7888

$-33.7139$

$-34.75$

$-22.26$

$-41.883$

$-37.8667$

138.0667
160.0854

160.0854

118.0667

141.0833

145.7

145.7

179.157
142.3667

121.58

146.7667

142.2667

160.0854
148.5167

148.5167

151.0303

150.5167

148.5

149.5567

177.88

153.4107

178.53

134.52

145.44

145.44
150.2222

134.57

134.52
134.603

134.603

102.93

145.8333

146.9472

142.3667

148.58

146.1667

146.3

170.2167

134.54

120.54

120.54

112.05
150.6774

150.6774
141.13

141.13

145.559

145.2189

145.7031 lacustrine micro, pollen slide

micro, pollen slide

11.69

micro, pollen slide

7.27

Builth et al., 2008

Longmore et al., 1986; Luly et al., 1986;

Luly, 1993, 1998

Longmore et al., 1986; Luly et al., 1986;

Luly, 1993, 1998

Harle et al., 2002

Hope, 2009

Haberle 1996

Hooley et al. 1980

Wang et al, 1999

Head, 1988

Kershaw et al., 2007

Kershaw, 1983

Hope et al., 2009
D'Costa et al., 1989

. Stevenson, unpublished dat

Robertson, 1986

Thevenon et al., 2004

Haberle, 1996

Kemp, 1993

Ladd et al., 1992

Devoy et al., 1994

Robbie, 1998

Worthy et al., 2005

G. Hope, unpublished data

Petherick et al., 2008

Hope et al., 2009

Athens and Ward, 2005

Hope, 2009

Van de Geer et al., 1989

halson, 1991

Athens and Ward, 2005

Athens and Ward, 2005

Athens and Ward, 2005

Athens and Ward, 2005

Penny and Kealhofer, 2004

Haberle and Ledru, 2001

McKenzie, 2002

D'Costa et al., 1989

Rogers and Hope, 2006

McKenzie, 2002

Moss and Kershaw, 2000

Vandergoes et al., 2005;

Newnham et al., 2007b

thens and Ward, 2005

Stevenson et al., 2009

Stevenson et al., 2009

Anshari et al., 2001

Chalson, 1991

Cupper, 2005, 2006

Stevenson, 1998

Colhoun, 1992

Pittock, 1989

McKenzie, 2002

3.30

(continued on next page) 


\begin{tabular}{|c|c|c|c|c|c|c|c|c|}
\hline Site Name & Latitude $\left({ }^{\circ}\right)$ & Longitude $\left({ }^{\circ}\right)$ & Elevation (m) & Site type & Charcoal methods & $\begin{array}{l}\text { Record length } \\
\text { (age ka) }\end{array}$ & $\begin{array}{l}\text { Resolution } \\
\text { (samples/ka) }\end{array}$ & References \\
\hline Quambie Lagoon & -12.5 & 131.17 & 20 & lacustrine & macro, sieved & 6.54 & 17.59 & J. Stevenson, unpublished data \\
\hline Queens Swamp Core QS 3 & -33.9002 & 150.5916 & 665 & mire & macro, sieved & 10.67 & 22.21 & S. Mooney, unpublished data \\
\hline Rawa Danau & -6.1833 & 105.9667 & 100 & lacustrine & micro, pollen slide & 16.59 & 1.87 & Haberle and Ledru, 2001 \\
\hline Redhead Lagoon & -32.9944 & 151.7208 & 65 & lacustrine & micro, pollen slide & 79.79 & 1.25 & Williams, 2005 \\
\hline Rennix Gap & -36.22 & 148.3 & 1570 & terrestrial & micro, pollen slide & 11.94 & 3.02 & G. Hope, unpublished data \\
\hline Ringarooma Humus Site 1 & -41.3 & 147.6167 & 885 & terrestrial & micro, pollen slide & 0.20 & 110.00 & Dodson et al., 1998 \\
\hline Ringarooma Humus site 2 & -41.3 & 147.6167 & 885 & terrestrial & micro, pollen slide & 0.32 & 53.13 & Dodson et al., 1998 \\
\hline Ringarooma River site I & -41.3 & 147.6167 & 885 & terrestrial & micro, pollen slide & 0.39 & 56.24 & Dodson et al., 1998 \\
\hline Ringarooma River Site II & -41.3 & 147.6167 & 885 & terrestrial & micro, pollen slide & 0.28 & 61.40 & Dodson et al., 1998 \\
\hline Rock Arch Swamp & -34.3 & 150.39 & 575 & mire & micro, pollen slide & 10.87 & 1.20 & Hope, 2005a \\
\hline Rotten Swamp (high-res study) & -35.7 & 148.8833 & 1445 & mire & micro, pollen slide & 6.31 & 56.74 & Hope and Clark, 2008 \\
\hline Rotten Swamp Core 4 & -35.7 & 148.8833 & 1445 & mire & micro, pollen slide & 11.41 & 1.58 & Clark, 1986 \\
\hline Ryans Swamp & -35.09 & 150.39 & 8 & mire & micro, pollen slide & 4.45 & 6.29 & Radclyffe, 1993 \\
\hline Saint Louis Lac & -22.23 & 166.55 & 5 & mire & micro, pollen slide & 7.19 & 8.63 & Stevenson, 2004 \\
\hline Sapphire Swamp & -30.0341 & 151.5604 & 1260 & mire & micro, pollen slide & 0.35 & 62.86 & Dodson et al., 1986 \\
\hline Sari & -16.63 & 179.5 & 67 & mire & micro, pollen slide & 6.53 & 3.52 & Hope et al., 2009 \\
\hline Snobs Creek & -37.558 & 145.928 & 775 & mire & micro, pollen slide & 15.32 & 2.02 & McKenzie, 1997 \\
\hline Snowy Flats & -35.54 & 148.47 & 1618 & mire & micro, pollen slide & 9.18 & 2.72 & Hope et al., 2005 \\
\hline Soleve & -17.25 & -179.49 & 2 & mire & micro, pollen slide & 7.16 & 2.93 & Clark and Hope, 1997 \\
\hline Solomons Jewel Lake & -41.8 & 146.2667 & 1185 & lacustrine & micro, pollen slide & 4.85 & 10.10 & Dodson, 2001 \\
\hline Sondambile & -6.3453 & 147.1116 & 2850 & lacustrine & macro, sieved & 1.34 & 243.12 & Haberle et al., 2005 \\
\hline South Salvation Creek Swamp & -33.6326 & 151.2587 & 132 & mire & micro, pollen slide & 4.78 & 4.19 & Kodela and Dodson, 1988 \\
\hline Stockyard Swamp, Hunter Island & -40.55 & 144.75 & 65 & mire & micro, pollen slide & 5.83 & 2.40 & Hope, 1999 \\
\hline Storm Creek & -37.45 & 145.8 & 1177 & Mire & micro, pollen slide & 29.33 & 1.40 & McKenzie, 1997 \\
\hline Sundown Swamp & -41.1667 & 144.6667 & 10 & mire & micro, pollen slide & 4.15 & 2.65 & Hope, 1999 \\
\hline Supulah Hill & -4.1167 & 138.9667 & 1580 & mire & micro, pollen slide & 39.00 & 0.95 & Haberle et al., 1991; Hope, 1998 \\
\hline Tadpole Swamp & -38.1317 & 145.2756 & 60 & mire & micro, pollen slide & 10.24 & 1.85 & Aitken and Kershaw, 1993 \\
\hline Tagamaucia & -16.49 & -179.56 & 820 & lacustrine & micro, pollen slide & 16.36 & 3.79 & Hope, 1996a \\
\hline Talita Kupai & -10.1 & 142.12 & 33 & coastal & macro, sieved & 2.41 & 61.72 & Rowe, 2006a \\
\hline Tea Tree Swamp Core DRA & -37.2167 & 148.8333 & 900 & mire & micro, pollen slide & 0.03 & 490.20 & Gell et al., 1993 \\
\hline Tea Tree Swamp Core DRE & -37.2167 & 148.8333 & 900 & mire & micro, pollen slide & 0.52 & 29.12 & Gell et al., 1993 \\
\hline Tea Tree Swamp Core DRN-A & -37.2167 & 148.8333 & 900 & mire & micro, pollen slide & 0.54 & 126.80 & Gell et al., 1993 \\
\hline Tiam Point & -10.12 & 142.18 & 3 & coastal & micro, pollen slide & 7.70 & 6.62 & Rowe, 2006a, 2007 \\
\hline Tiger Snake Swamp & -38.1313 & 145.2758 & 60 & mire & micro, pollen slide & 8.41 & 2.73 & Aitken and Kershaw, 1993 \\
\hline Tom Burns (D-section Core) & -37.3833 & 145.8167 & 1075 & Mire & micro, pollen slide & 12.07 & 2.57 & McKenzie, 1997 \\
\hline Tom Burns (Missen Core) & -37.3833 & 145.8167 & 1075 & Mire & micro, pollen slide & 31.56 & 0.48 & McKenzie, 1997 \\
\hline Tom Gregory Swamp & -35.38 & 148.49 & 1024 & mire & micro, pollen slide & 12.39 & 1.29 & Hope, 2005b \\
\hline Tugupugua & -5.6691 & 142.6108 & 2300 & mire & micro, pollen slide & 18.42 & 3.42 & Haberle and Ledru, 2001 \\
\hline Tyrendarra Swamp & -38.1986 & 141.7626 & 13 & marsh & micro, pollen slide & 31.63 & 1.04 & Builth et al., 2008 \\
\hline Voli Voli & -18.16 & 177.485 & 2 & mire & micro, pollen slide & 5.87 & 7.15 & Dickinson et al., 1998 \\
\hline Vunimoli & -18.22 & 177.88 & 251 & mire & micro, pollen slide & 4.75 & 2.11 & Hope et al., 1996, 1999 \\
\hline Waikaremoana & -38.45 & 177.02 & 582 & lacustrine & micro, pollen slide & 1.65 & 9.11 & Newnham et al., 1998 \\
\hline Waitabu & -18.23 & -178.781 & 43 & other & micro, pollen slide & 1.71 & 18.16 & Latham et al., 1983 \\
\hline Wanda & -2.33 & 121.23 & 440 & mire & micro, pollen slide & 49.30 & 1.24 & Hope, 2001 \\
\hline Wanum & -6.6365 & 146.7986 & 35 & lacustrine & macro, sieved & 3.89 & 72.92 & Haberle et al., 2005 \\
\hline Warrananga & -33.97 & 141.56 & 22 & lacustrine & micro, pollen slide & 10.96 & 4.93 & Cupper, 2005, 2006 \\
\hline Warrimoo Swamp & -33.7226 & 150.6162 & 195 & mire & micro, pollen slide & 4.63 & 6.27 & Chalson, 1991 \\
\hline Waruid & -10.4 & 142.09 & 5 & coastal & macro, sieved & 7.20 & 27.10 & Rowe, 2006a, 2007 \\
\hline Wet Lagoon & -34.47 & 149.25 & 700 & mire & micro, pollen slide & 2.30 & 12.17 & Dodson, 1986 \\
\hline Whitehaven Swamp & -20.3 & 148.9 & 45 & mire & micro, pollen slide & 8.04 & 6.60 & Genever et al., 2003 \\
\hline Wildes Meadow Swamp & -34.6208 & 150.5111 & 670 & mire & micro, pollen slide & 3.62 & 3.04 & Kodela, 1996 \\
\hline Wilson Bog & -34.97 & 138.69 & 425 & mire & macro, sieved & 7.18 & 13.23 & Buckman et al., 2009 \\
\hline Wingecarribee Swamp W2 & -34.5666 & 150.5166 & 685 & mire & macro, sieved & 3.50 & 124.68 & de Montford, 2008 \\
\hline Worimi Swamp & -32.5166 & 152.3333 & 8 & mire & macro, sieved & 2.46 & 36.53 & Mooney and Maltby, 2006 \\
\hline Wyelangta & -38.6472 & 143.4614 & 450 & fluvial & micro, pollen slide & 223.93 & 0.23 & McKenzie and Kershaw, 2000 \\
\hline Xere Wapo B & -22.29 & 166.97 & 220 & lacustrine & micro, pollen slide & 126.04 & 0.52 & Stevenson and Hope, 2005 \\
\hline
\end{tabular}



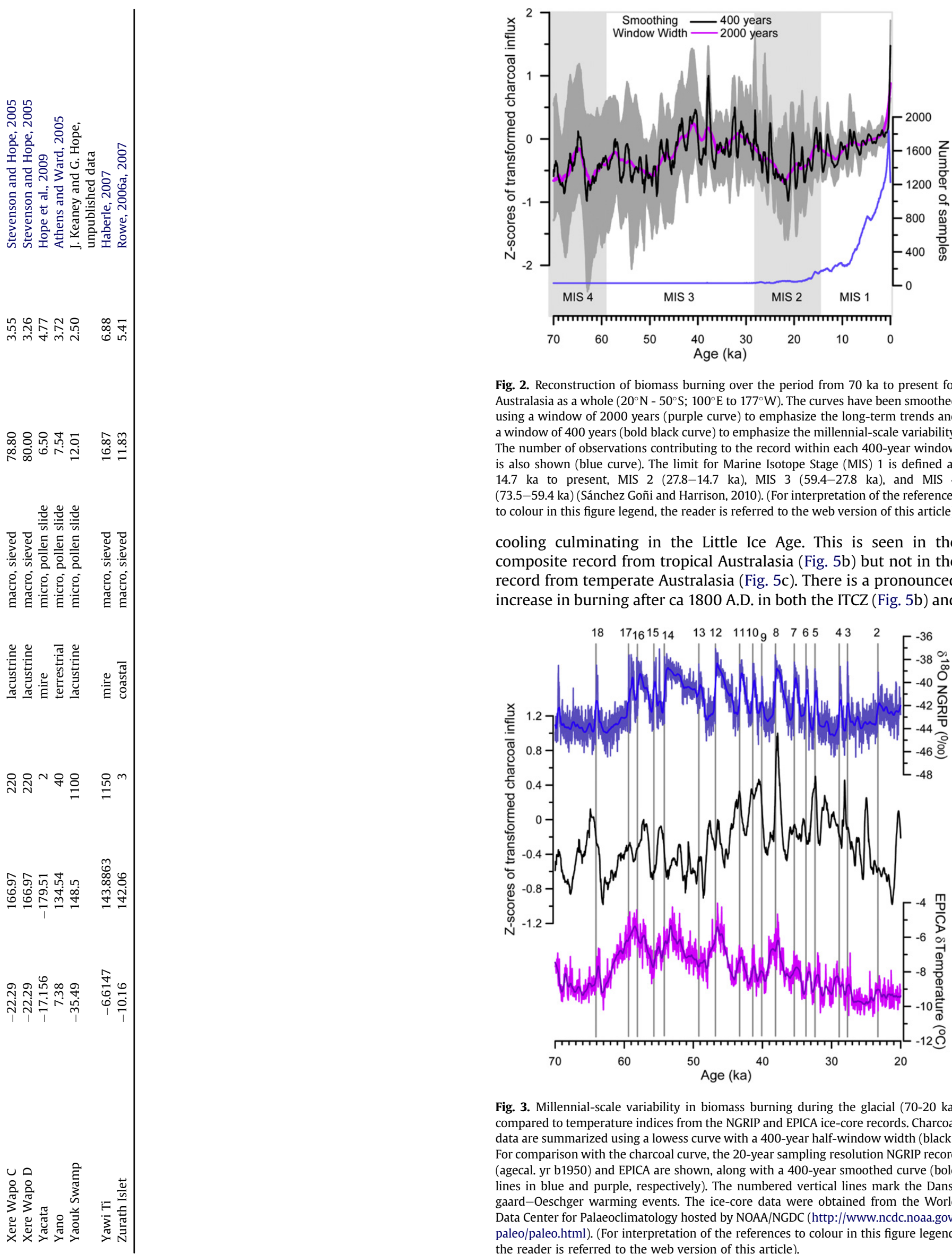

Fig. 2. Reconstruction of biomass burning over the period from 70 ka to present for Australasia as a whole $\left(20^{\circ} \mathrm{N}-50^{\circ} \mathrm{S} ; 100^{\circ} \mathrm{E}\right.$ to $\left.177^{\circ} \mathrm{W}\right)$. The curves have been smoothed using a window of 2000 years (purple curve) to emphasize the long-term trends and a window of 400 years (bold black curve) to emphasize the millennial-scale variability. The number of observations contributing to the record within each 400-year window is also shown (blue curve). The limit for Marine Isotope Stage (MIS) 1 is defined as $14.7 \mathrm{ka}$ to present, MIS 2 (27.8-14.7 ka), MIS 3 (59.4-27.8 ka), and MIS 4 (73.5-59.4 ka) (Sánchez Goñi and Harrison, 2010). (For interpretation of the references to colour in this figure legend, the reader is referred to the web version of this article).

cooling culminating in the Little Ice Age. This is seen in the composite record from tropical Australasia (Fig. 5b) but not in the record from temperate Australasia (Fig. 5c). There is a pronounced increase in burning after ca 1800 A.D. in both the ITCZ (Fig. 5b) and

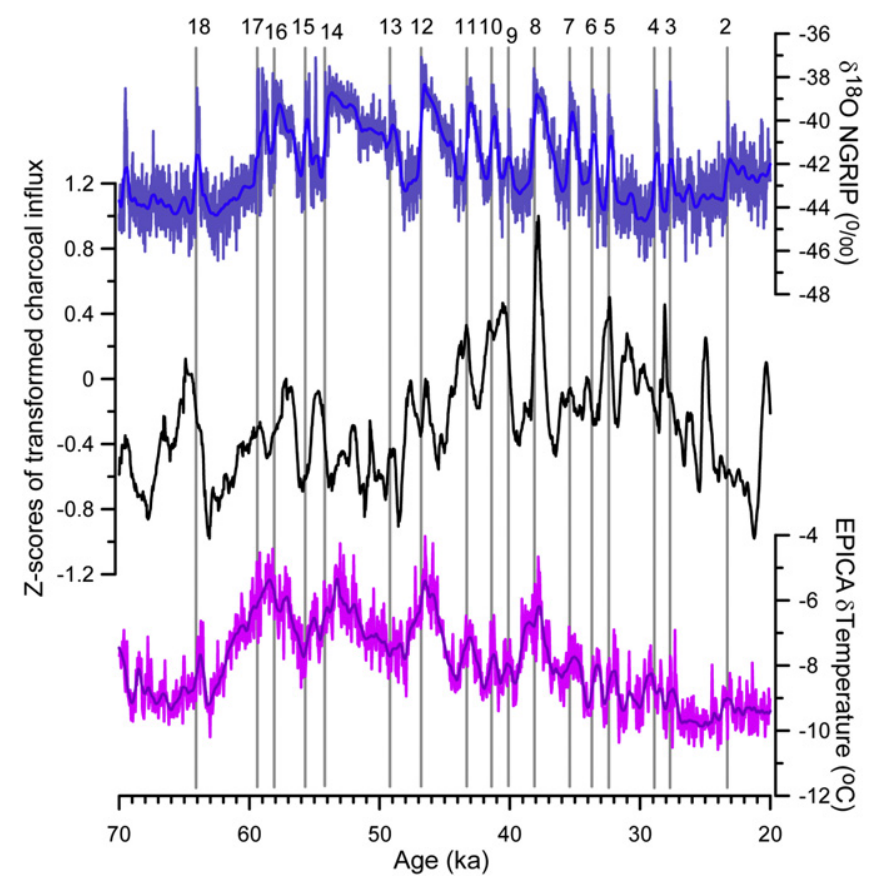

Fig. 3. Millennial-scale variability in biomass burning during the glacial (70-20 ka) compared to temperature indices from the NGRIP and EPICA ice-core records. Charcoal data are summarized using a lowess curve with a 400-year half-window width (black). For comparison with the charcoal curve, the 20-year sampling resolution NGRIP record (agecal. yr b1950) and EPICA are shown, along with a 400-year smoothed curve (bold lines in blue and purple, respectively). The numbered vertical lines mark the Dansgaard-Oeschger warming events. The ice-core data were obtained from the World Data Center for Palaeoclimatology hosted by NOAA/NGDC (http://www.ncdc.noaa.gov/ paleo/paleo.html). (For interpretation of the references to colour in this figure legend, the reader is referred to the web version of this article). 

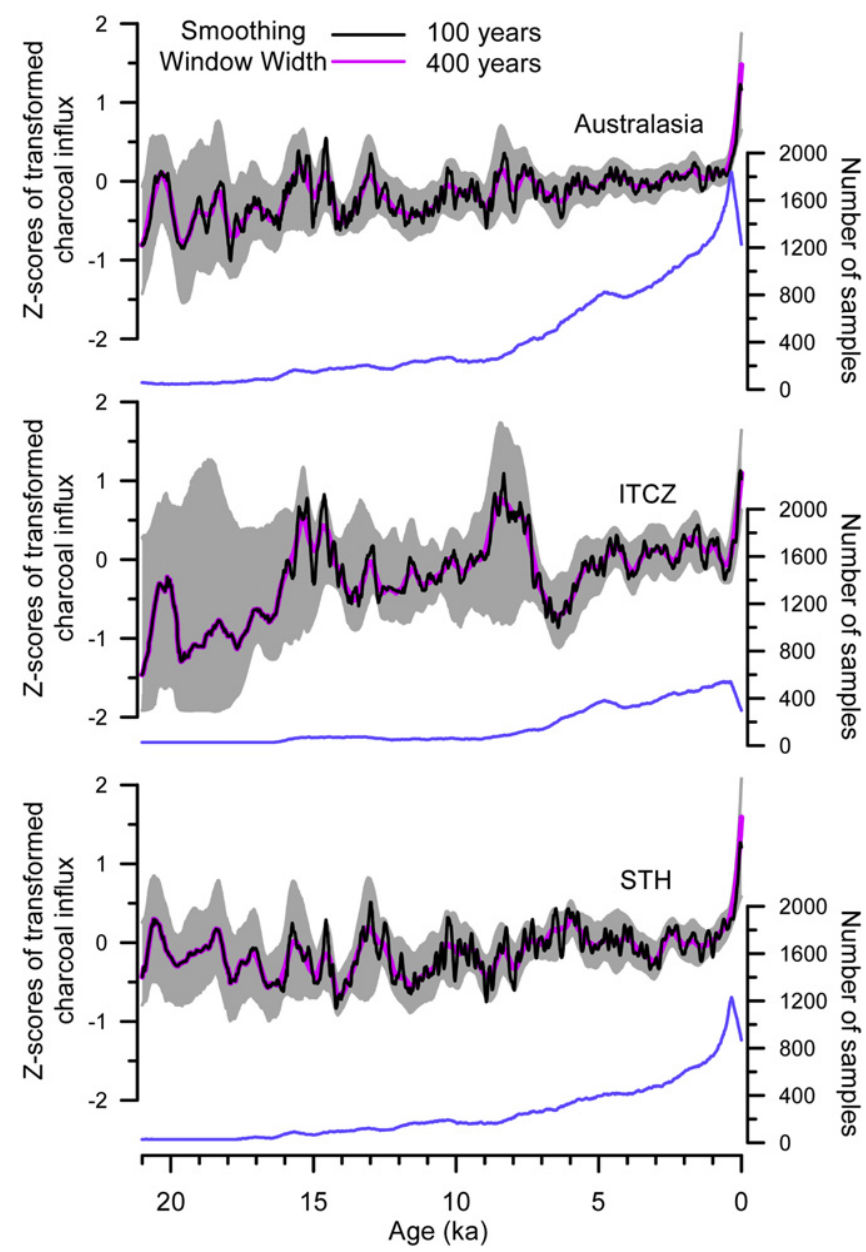

Fig. 4. Reconstruction of biomass burning for the last 21 ka for Australasia as a whole $\left(20^{\circ} \mathrm{N}-50^{\circ} \mathrm{S}, 100^{\circ} \mathrm{E}-177^{\circ} \mathrm{W}\right)$, the belt corresponding to the modern Inter-tropical Convergence Zone (ITCZ: $20^{\circ} \mathrm{N}-20^{\circ} \mathrm{S}, 100^{\circ} \mathrm{E}$ to $177 \mathrm{~W}$, broadly the tropical region) and the modern sub-tropical high pressure belt (STH: $25^{\circ} \mathrm{S}-45^{\circ} \mathrm{S}, 100^{\circ} \mathrm{E}$ to $177^{\circ} \mathrm{W}$, broadly temperate Australasia). The curves have been smoothed using a window of 400 years (purple curve) and a window of 100 years (bold black curve) to emphasize the centennial-scale variability. The bootstrapped confidence intervals are based on a $400-$ year smoothing of the curves. The number of observations contributing to the record within each 400-year window is also shown (blue curves). (For interpretation of the references to colour in this figure legend, the reader is referred to the web version of this article).

STH regions (Fig. 5c), and this increase is also seen in the composite record of the Australian mainland (Fig. 5d). The upturn is delayed compared to the timing of the increase in global biomass burning which occurred ca 1750 A.D (Marlon et al., 2008). Although European colonization of Australia may have been responsible for the upturn seen on the mainland, and in the composite regional curve, it is more difficult to invoke this explanation for the record from tropical Australasia (Fig. 5b) which suggests that any anthropogenic influence was exacerbated by changes in climate and vegetation productivity associated with the post-industrial increase in atmospheric $\mathrm{CO}_{2}$ concentration. The curves for all of the regions show reduced fire during the last $\mathrm{ca}$. 50 years, and this is despite the widespread use of prescribed burning as a means of fire control in much of Australia. The observed reduction in biomass burning in Australasia occurs a few decades after the global reduction in biomass burning which Marlon et al. (2008) attribute to increased landscape fragmentation and fire suppression. There are a number of reasons why the last fifty or so years of the record might be less tightly constrained, including issues with sampling, problems with

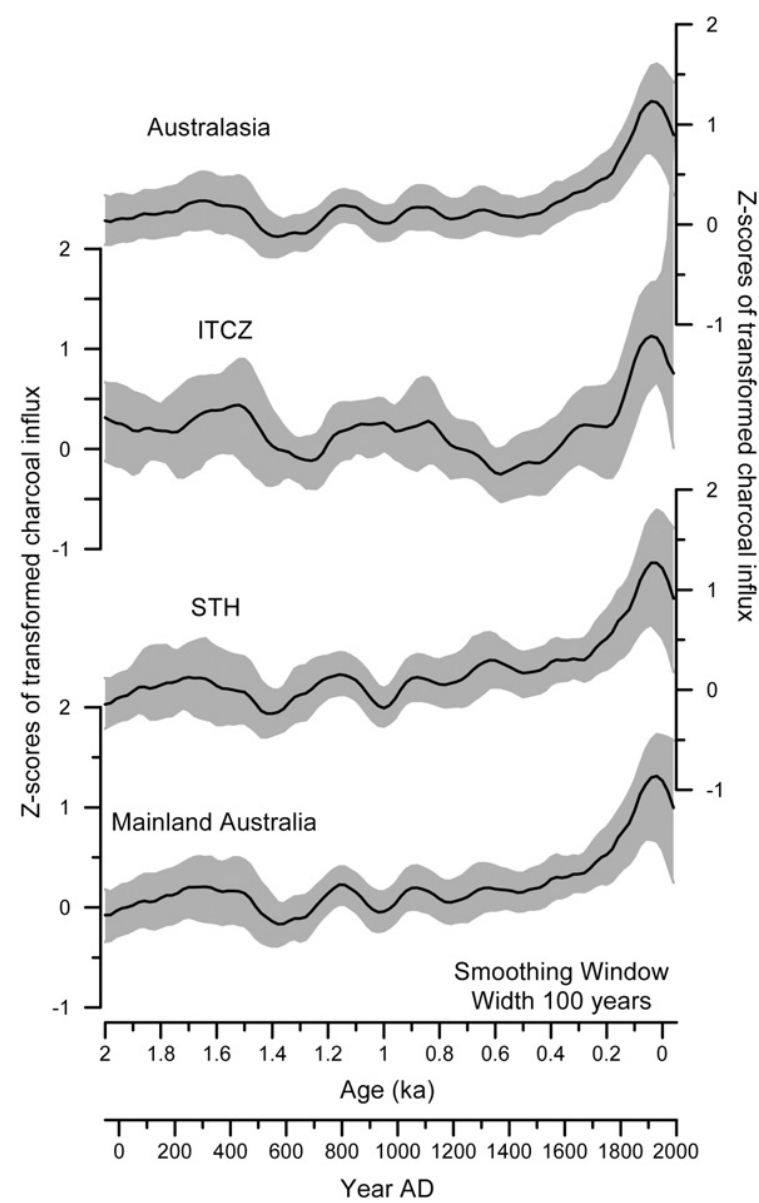

Fig. 5. Reconstruction of biomass burning for the last 2 ka for Australasia as a whole $\left(20^{\circ} \mathrm{N}-50^{\circ} \mathrm{S}, 100^{\circ} \mathrm{E}-177^{\circ} \mathrm{W}\right)$, the belt corresponding to the modern Inter-tropical Convergence Zone (ITCZ: $20^{\circ} \mathrm{N}-20^{\circ} \mathrm{S}, 100^{\circ} \mathrm{E}$ to $177 \mathrm{~W}$, broadly the tropical region), the modern sub-tropical high pressure belt ( $\mathrm{STH}$ : $25^{\circ} \mathrm{S}-45^{\circ} \mathrm{S}, 100^{\circ} \mathrm{E}$ to $177^{\circ} \mathrm{W}$, broadly temperate Australasia), and for all sites on the Australian mainland. The curves have been smoothed using a window of 100 years (bold black curve).

dating and the potential impact of human activities on catchment processes and sedimentation (Gale, 2009). Nevertheless, there are over 140 sites contributing to the record during the 20th century and 168 sites recording the last two centuries, and thus the recent downturn in fire appears to be a robust feature of the record. Furthermore, Marlon et al. (2008) demonstrate (in their supporting information) that the rapid increase in biomass burning followed by an abrupt decrease cannot be explained by simple sedimentation rate variations at the top of cores.

\subsection{Spatial patterns in biomass burning since the Last Glacial Maximum}

Most of the sites in our data set are concentrated in eastern, particularly southeastern Australia (Fig. 1a). Nevertheless, there are spatial patterns in the changes in biomass burning that are worth exploring. Here (Fig. 6), we present maps of the average z-scores for key intervals since the LGM to illustrate some of the regional patterns of change: positive $z$-scores indicate more biomass burning and negative z-scores less biomass burning than the longterm average for the base period (21-0.2 ka).

The LGM (Fig. 6a) was characterized by low biomass burning in the tropics and over most of Australia. A few sites in southeastern Australia and one site on the South Island of New Zealand show higher-than-average $\mathrm{z}$-scores. There is considerable millennial- 

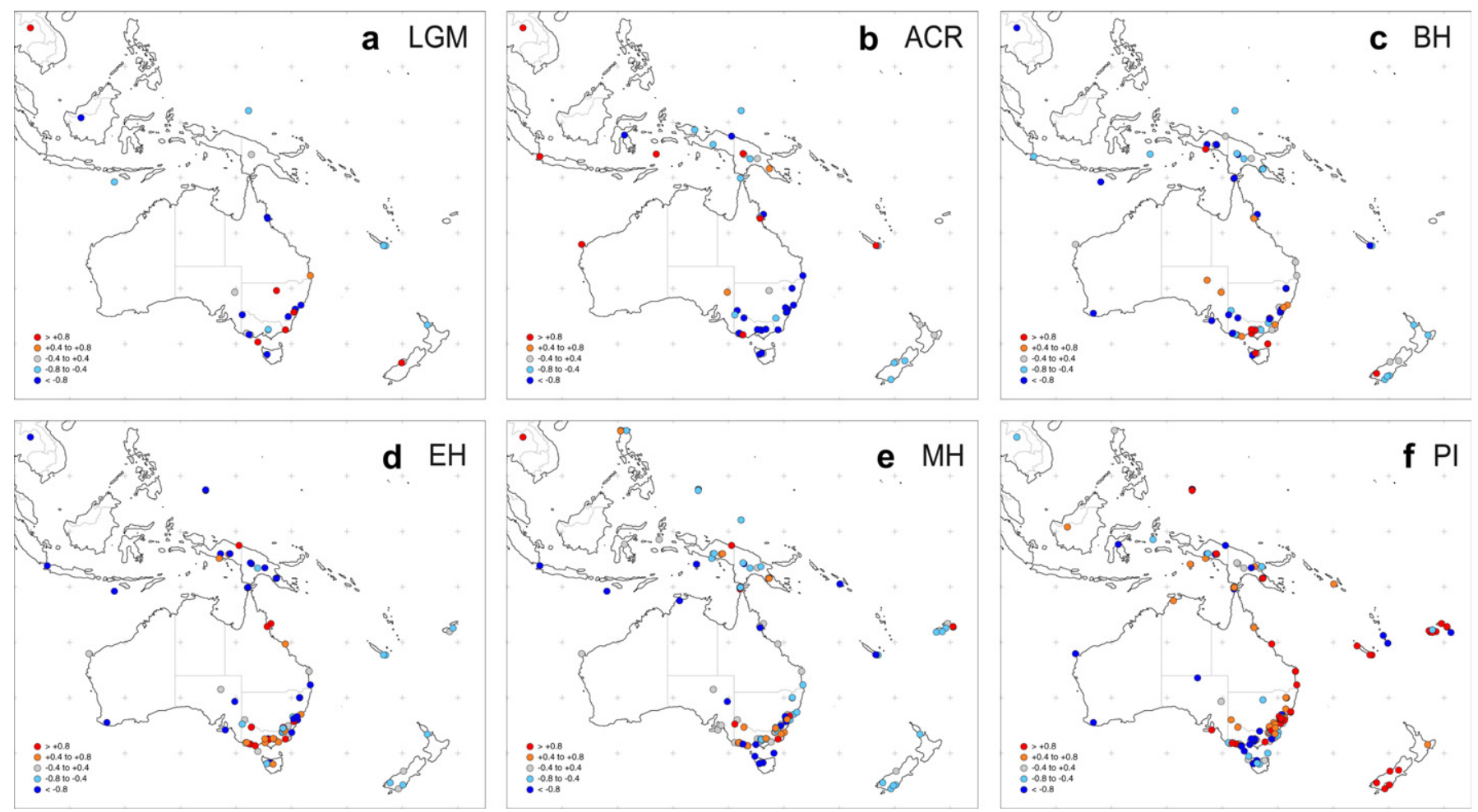

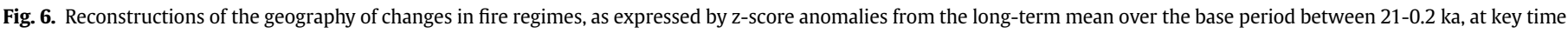

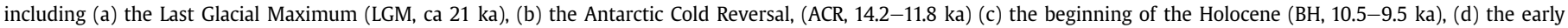
Holocene ( $\mathrm{EH}, 8.5-7.5 \mathrm{ka})(\mathrm{e})$ the mid-Holocene, $(\mathrm{MH}, 6-5 \mathrm{ka})$, (f) the pre-industrial period (PI, 0.7-0.2 ka).

scale variability superimposed on the long-term increase in biomass burning after the LGM (Fig. 4) and this is reflected in the mapped patterns, with individual sites showing more/less fire in succeeding 1000-yr intervals (not shown). However, a more coherent spatial pattern is established during the Antarctic Cold Reversal (ACR) (Fig. 6b), with low biomass burning in southeastern Australia and New Zealand contrasting with high biomass burning at many sites in the tropics. In contrast, the beginning of the Holocene is marked by high biomass burning in southeastern Australia and low biomass burning in the tropics (Fig. 6c).

The most marked spatial feature of the Holocene record is the opposition in the temperate latitudes between sites in southernmost southeastern Australia (including Tasmania) and southeastern NSW (including the interior). In the early Holocene, here illustrated by the interval centered on $8 \mathrm{ka}$ (Fig. 6d), the southern region is characterized by high biomass burning and the region further north by low biomass burning. By the mid-Holocene, here illustrated by the interval centered on $5.5 \mathrm{ka}$ (Fig. 6e), sites in Tasmania and the southernmost tip of the continent show less biomass burning and many sites further north show increased biomass burning. The pattern has reversed again by the late Holocene (not shown). The pre-industral era (0.2-0.7 ka, Fig. 6f) is characterized by high biomass burning along the east coast of Australia, in New Zealand and over much of the tropics. However, Tasmania and the southernmost part of southeastern Australia, and the limited number of sites from western Australia, show less biomass burning than average.

Although there are robust, large-scale patterns in changes in biomass burning through time, the changes within any one region through time are complex; adjacent sites can show differences even in the sign of the change at particular times. This may, in part, be a function of the quality of the data and of individual age models. It is also in the nature of wildfires, which are spatially disjunct, influenced by topography and the changing nature of the vegetation itself, and conditioned by timing of previous fires.

\subsection{Fire and humans during the late Pleistocene}

Smith et al. (2008) have used a data set of 971 radiocarbon ages from 286 archaeological sites in arid Australia (AustArch1) to illustrate the overall trend in archaeological site incidence by summing the probability density functions of individual radiocarbon ages, which they suggest can be interpreted as a first approximation of human activity and population history through time. Smith et al. (2008) stress the preliminary nature of this record, but nevertheless the compilation allows us to explore the relationship between an approximate measure of human activity and the charcoal-derived record of fire from Australia (Fig. 7). Although there are other compilations of archaeological information from Australia (e.g. Lourandos and David, 1998; Lourandos and David, 2002; Turney and Hobbs, 2006), the AustArch1 data set is the most comprehensive and the raw data are available in a format which allows reanalysis.

There are relatively few archaeological dates, and little structure in the probability density curve, prior to $c a .20 \mathrm{ka}$. In contrast, there are large changes in fire on millennial timescales between 40-20 ka. The lack of congruence between the archaeological and fire records during this period suggests that the changes in fire activity do not reflect changes in human activity. Smith et al. (2008) identified six intervals of increased "human activity" during the past $20 \mathrm{ka}$, including a major increase in the late Holocene. Some of the reconstructed peaks in human activity correspond to peaks in fire (e.g. 7-8 ka). However, other peaks in human activity correspond to troughs in biomass burning (e.g. 5-4.5 ka) and there are peaks in biomass burning that have no correspondence with changes in human activity (e.g. at $13 \mathrm{ka}$ and $9.5 \mathrm{ka}$ ). Most importantly, the 


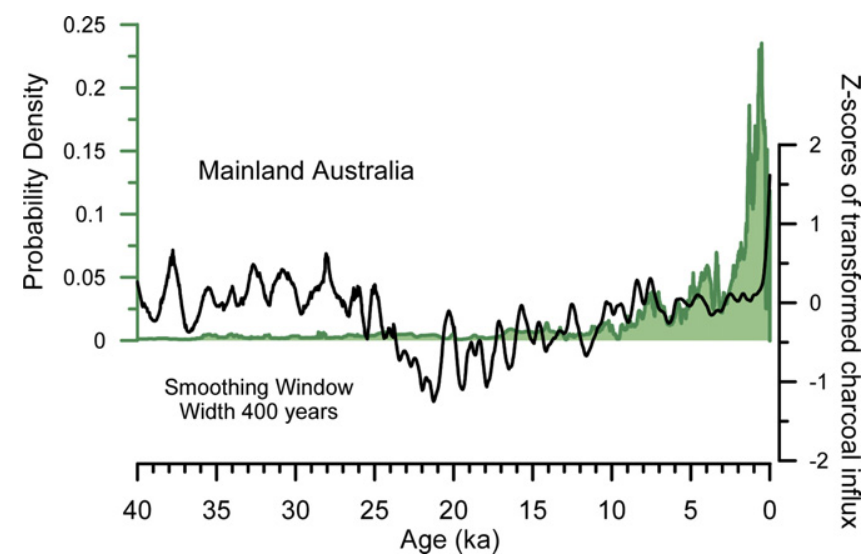

Fig. 7. Comparison of the composite charcoal curve for sites from the Australian mainland over the last $40 \mathrm{ka}$ (black curve) compared to probability density estimates of human populations based on radiocarbon-dated archaeological records (green infilled curve). Population data from the AustArch Database (see Smith et al., 2008 for fuller description of the methodology). (For interpretation of the references to colour in this figure legend, the reader is referred to the web version of this article).

major inferred increase in population during the late Holocene ('intensification') is not accompanied by an increase in fire. Thus, this comparison does not support the hypothesis that changes in post-glacial fires in Australia were caused by humans.

\section{Discussion and conclusions}

We have presented an analysis of the long-term changes in fire regimes across Australasia based on a comprehensive synthesis of over 200 charcoal records from the region. Despite the supposed importance of fire for Australasian vegetation (Bowman, 2000; Bradstock et al., 2002), the long-standing arguments about the role of Aboriginal colonisation on fire regimes (e.g. Singh et al., 1981; Horton, 2000; Flannery, 1994; Kershaw et al., 2002; Black and Mooney, 2006), and the uncertainty about the impact of climate change on fire regimes and fire-related disasters (Chapman, 1999; Williams et al., 2001; Cary, 2002; Bushfire Co-operative Research Centre, 2006; Russell-Smith et al., 2007), there have been relatively few analyses of the palaeo-record of fire at a continental scale.

Previous reviews (see e.g. Singh et al., 1981; Kershaw et al., 2002; Lynch et al., 2007) have been based on comparatively few sites and the assumption that iconic records are characteristic of much broader geographic regions. Although there are obvious spatial gaps in the data set used in this analysis, and some uncertainty associated with pooling records with age models of very different quality, we have been able to document robust changes in fire regime through time for which there are physically plausible explanations. The analysis presented here is preliminary in nature: much more could be done to explore the existing data set. Nevertheless, by demonstrating the potential of large-scale syntheses of charcoal data to shed light on the environmental history of the continent we hope to encourage the collection of additional, highquality data from the Australasian region.

On glacial-interglacial timescales, changes in fire regimes closely follow global temperature: biomass burning is reduced during cold glacial or stadial intervals (MIS 4, MIS 2) and increased during warmer interglacial or interstadial intervals (MIS 3, the Holocene). This finding is consistent with global analyses (e.g. Power et al., 2008; Daniau et al., 2010) and presumably reflects the strong control of vegetation productivity on the availability of fuel (Harrison et al., 2010). Although we have confined our detailed analyses to the past $70 \mathrm{ka}$, we see no evidence for the expression of long-term aridification of Australia (see e.g. Lynch et al., 2007) in the charcoal records of fire regimes. The length of this interval of increasing aridity cited in the literature is somewhat vague, but we see no evidence for a long-term trend in biomass burning superimposed on the glacial-interglacial pattern.

The charcoal records show considerable millennial-scale variability during the glacial. Given the limited radiometric age control on these records, and the widespread use of correlation to assumed stage/event boundaries, it is unclear whether individual peaks in charcoal correlate with specific D-O warming events registered in the Greenland ice core and reflected in marine records from the North Atlantic. Nevertheless, the shape of the charcoal curves is more reminiscent of the temperature changes in Greenland than those recorded in Antarctica (Fig. 3) and, in conjunction with the number of events registered and the approximate temporal correlations of these events, suggests that Australasian fire regimes have co-varied with D-O cycles: with increased fire during warming events and Greenland Interstadials and reduced fire during Greenland Stadials. Millennial-scale variability has been identified in several individual records from Australasia (see e.g. Sikes et al., 2002; Turney et al., 2004; Vandergoes et al., 2005; Kershaw et al., 2007), although with the caveat that the dating was insufficient to determine whether this variability was in or out of phase with the D-O cycles. However, Muller et al. (2008) have stated explicitly that warmer intervals of the D-O cycles are associated with dry conditions at Lynch's Crater.

EPICA Community Members (2006) have demonstrated that the temperature records from Greenland and Antarctica are out of phase, and this bi-polar temperature seesaw is attributed to the shutdown or slowdown of the thermohaline circulation (see Kageyama et al., 2010). However, the bi-polar seesaw hypothesis does not imply that the opposition in the direction of the change in temperature shown by the ice-core records is a pan-hemispheric phenomenon. In fact, decomposition of climate variability during the deglaciation (Shakun and Carlson, 2010), based on 104 highresolution palaeoclimate records, has shown that approximately $60 \%$ of the climate signal is common between the two hemispheres, while only $11 \%$ of the signal is associated with the bi-polar seesaw. Shakun and Carlson (2010) attributed the hemispheric synchroneity in climate to the influence of $\mathrm{CO}_{2}$, although presumably this argument would apply to all of the greenhouse gases. The greenhouse gas synchronisation of hemispheric climate changes likely operated during the whole of the glacial, where D-O variability was accompanied by changes in $\mathrm{CO}_{2}$ of the order of $15-20 \mathrm{ppm}$ and $\mathrm{CH}_{4}$ values of the order of about $100 \mathrm{ppm}$, or about $20 \%$ and $50 \%$ of their glacial-interglacial range, respectively (Ahn and Brook, 2008). There are other mechanisms that could extend a northern-hemisphere climate signal into the southern hemisphere, through changes in atmospheric circulation that accompany THC shutdown, as shown by freshwater-forcing experiments (Stouffer et al., 2006: Muller et al., 2008; Kageyama et al., 2009, 2010). Thus, the observation that Australasian fire regimes display some coherence with millennial-scale northern-hemisphere climate variability is mechanistically credible (Clark et al., 2002; Muller et al., 2008; Daniau et al., 2010). Furthermore, Southern Hemisphere vegetation records show millennial-scale variability (see e.g. Hessler et al., 2010; Harrison and Sanchez Goni, 2010), although the vegetation response appears to be more muted than shown in European records (Fletcher et al., 2010) it is nevertheless in phase with D-O events (Harrison and Sanchez Goni, 2010).

The pre-40 ka composite biomass-burning curve for Australasia is based on 25 individual records and this value rises to 38 sites by $30 \mathrm{ka}$. There is clearly a need for more long records, sampling a wider geographic range of regional climates. Charcoal records from marine cores are providing an increasingly detailed view of 
changes in fire regimes during the glacial (see e.g. Beaufort et al., 2003; Thevenon et al., 2004; Daniau et al., 2007, 2009) with the added advantage that these records can be linked, through isotope stratigraphy, directly to changes in sea-surface temperatures and to changes recorded in ice cores. Marine records could be more widely exploited for charcoal analysis in the Australasian region.

Low biomass burning was characteristic of the LGM. Vegetation reconstructions show an expansion of xerophytic vegetation in southern Australia and of tropical deciduous broadleaf forest and woodland in the tropics (both mainland Australia, Papua and the islands of southeastern Asia) at the LGM (Pickett et al., 2004). Although the number of records included in this synthesis is limited, particularly for northern Australia, the interpretation is consistent with earlier reconstructions (see e.g. Markgraf et al., 1992; Harrison and Dodson, 1993) showing expansion of drought-tolerant vegetation during the LGM. Although superficially these changes could be interpreted as an expansion of more fire-prone vegetation, they have been interpreted as indicating colder and drier conditions. Indeed, Williams et al. (2009) argue for a reduction in precipitation of ca $30-50 \%$ in the tropics and a significant reduction in winter precipitation across the temperate zone, accompanied by reductions in temperature of ca $3-8{ }^{\circ} \mathrm{C}$. The observed reduction in biomass burning must reflect a significant reduction in fuel availability under cold, dry conditions, sufficient to offset the increase in aridity and also the stronger winds that have been adduced from dune evidence (e.g. Hesse et al., 2004).

Previous syntheses of palaeoenvironmental data from Australia have identified the late glacial as an interval of aridity, more pronounced than during the LGM and certainly than during the Holocene (see e.g. Harrison and Dodson, 1993; Kershaw et al., 2003; Williams et al., 2009). The charcoal records show considerable spatial and temporal variability during the deglaciation. There is no evidence for dry conditions persisting for several thousand years, as postulated by earlier syntheses. However, the charcoal records do appear to show a reduction in biomass burning during the ACR. The ACR has been identified in palaeoenvironmental records from southeastern Australia and New Zealand (see e.g. Barrows et al., 2007; Calvo et al., 2007; Williams et al., 2009), although the recognition that this climate event could have had an impact on fire regimes at a continental scale is new.

Vegetation reconstructions (see e.g. Harrison and Dodson, 1993; Pickett et al., 2004) indicate that the mid-Holocene vegetation patterns of Australia were very similar to those typical of the preindustrial (and pre-European) era. Despite the lack of vegetation change, Lynch et al. (2007) indicate that the interval between 7 $5 \mathrm{ka}$ (which they call the "climatic optimum") was one of reduced biomass burning in southeastern Australia. They do not specify which records this assertion is based on, and their conclusion is certainly not supported by the records presented here: most sites in southeastern Australia show considerably higher-than-average levels of fire at $6 \mathrm{ka}$, although sites in the interior (and the limited number of sites in western and northern Australia) show less-thanaverage biomass burning.

We have identified a tendency for sites in the southernmost part of southeastern Australia to show an opposite pattern of change in biomass burning during the Holocene from those further north and along the east coast. This spatial differentiation was also identified by Pickett et al. (2004) in their reconstruction of mid-Holocene vegetation shifts. They argued that sites in the far south show a shift towards more moisture-stressed vegetation in the mid-Holocene, while sites in the Snowy Mountains, on the Southern Tablelands and east of the Great Dividing Range have more moisturedemanding vegetation in the mid-Holocene than today. These shifts in vegetation are consistent with the reconstructed shifts in biomass burning, with the former region characterized by more fire and the latter by less fire during the mid-Holocene.

Lynch et al. (2007) have argued that the pronounced increased in biomass burning after ca. 4 ka shown in the charcoal record from Lake Euramoo (Haberle, 2005), and assumed to represent a regional signal, reflects an increases in climate variability associated with ENSO. There is considerable evidence for an increase in ENSO strength and/ or variability after the mid-Holocene (see e.g. Rodbell et al., 1999; Tudhope et al., 2001; Andrus et al., 2002; Koutavas et al., 2002) but the onset of increased variability has been variously placed at $6.5 \mathrm{ka}$ (Black et al., 2008), $5 \mathrm{ka}$ (McGlone et al., 1992), $4 \mathrm{ka}$ (Shulmeister and Lees, 1995) and $3 \mathrm{ka}$ (Gagan et al., 2004) and reanalysis of the Lake Pallcacocha record (Rodbell et al., 1999) suggests that variations on the ENSO time scale persisted throughout the Holocene (Rodó and Rodriguez-Arias, 2004). Neither the composite nor regional charcoal records (Fig. 4) show a clear relationship between changes in ENSO (e.g. Moy et al., 2002) and patterns of biomass burning. This suggests the need for a re-evaluation of the relationship between biomass burning and changes in ENSO, over both late Holocene and longer timescales, taking into account that the sign of the relationship between biomass burning and ENSO may be different depending on the nature of the vegetation.

Lack of data has bedevilled previous interpretations of both the fire history and, more generally, the palaeoenvironmental history of Australasia. Reconstructed changes at individual sites have often been used to draw quasi-continental scale inferences about past climate and environmental changes. The fact that many of the conclusions drawn from such iconic sites are not supported by the large-scale data synthesis of charcoal records presented here argues for an urgent need to re-assess our understanding of the late Quaternary history of Australia.

Chronology has also been a contentious issue for the interpretation of palaeofire records from Australasia. Alternative age models have been proposed for several of the longer records included in this synthesis (see for e.g. Lynch's Crater: Kershaw, 1986; Turney et al., 2001, 2004; Kershaw et al., 2007; Muller et al., 2008; Lake George: Singh et al., 1981; Singh and Geissler, 1985; Fitzsimmons and Barrows, 2010). In the interpretation of individual records, the quality of the age model is of prime importance and reliance on assumed correlations with global events or stratigraphic boundaries (e.g. the MIS boundaries) precludes detailed analysis of the relationships between observed changes in fire and the global controls on regional climate. The strength of the technique used here of compositing multiple records is that it allows a robust assessment of the influence of individual records, and hence of the chronological uncertainties of each record, on the shape of the composite curve. By focusing on statistically robust features of the composite record, we are able to draw plausible inferences about the controls on these features. Clearly, improvement of the individual age models and increased reliance on radiometric dating would substantially enhance the amount of detail that could be extracted from the composite records. Nevertheless, our analyses show that there is considerable temporal structure in the fire records and it is highly unlikely that this structure could be generated by chance, particularly since the reconstructed changes in biomass burning through time are consistent with current understanding of past climate changes.

The simplest interpretation of the charcoal records is that climate, and climate-modulated changes in vegetation productivity and distribution, control fire regimes on centennial to multimillennial timescales. The observed changes in biomass burning can be plausibly explained by current understanding of the broadscale changes in climate over the last ca. 70 ka. However, there are many changes in the fire record for which we still lack a robust mechanistic explanation. As previous work has shown (e.g. 
Power et al., 2008), simplistic explanations of fire records in terms of single climate (or environmental) drivers are likely to be wrong. Similar changes in climate can produce changes in biomass burning of opposite sign depending on the state of the vegetation (van der Werf et al., 2008b; Harrison et al., 2010). For example, an increase in precipitation will tend to suppress fires in most forests but could lead to an increase in fire in areas where fuel is limited because it will support more plant growth. Coeval changes in temperature can offset the impact of changes in precipitation on vegetation productivity. Atmospheric $\mathrm{CO}_{2}$ concentrations influence vegetation productivity and distribution even without a change in climate (Harrison and Prentice, 2003; Prentice and Harrison, 2009). Finally, fire regimes are affected by the considerable heterogeneity of local climates. Sites in close geographic proximity may nevertheless have different climates, particularly with respect to rainfall seasonality. Shafer et al. (2005), for example, have shown that large-scale changes in atmospheric circulation lead to opposite changes in precipitation between sites in the same region depending on whether they experience a summer or winter-rainfall maximum and this in turn affects the response of the fire regime to these changes in atmospheric circulation (Millspaugh et al., 2004). Given all these competing influences, it is unlikely that interpretations of the charcoal record in terms of climate will be unequivocal. While speculations as to the causes of specific changes in fire regimes are interesting as a source of hypotheses, we would argue strongly for the combined use of observational data and modelling (see e.g. Webb et al., 1998; Harrison et al., 2003) to interpret the observed changes in fire regimes through time.

The influence of humans in modifying natural fire regimes has been a major feature of the interpretation of charcoal records from the Australasian region. The apparent coincidence of increases in sedimentary charcoal at iconic sites (Lake George, Lynch's Crater, Darwin Crater) with the arrival of Aboriginal people in Australia, has been attributed to anthropogenic fire (e.g. see Turney et al., 2004). This has led to circular arguments about the relationship between fire and people, including assertions that people arrived on the continent before the last glacial (Jackson, 1999; Singh et al., 1981). Changes in vegetation cover driven by anthropogenic modification of fire regimes have been explicitly invoked as a mechanism for causing aridification of Australia over the past 50-60 ka and for megafaunal extinctions (Miller et al., 2005). This causal association of anthropogenic fire with changes in climate, vegetation or fauna has remained seductive, despite several lines of contrary evidence. This evidence includes major changes in Late Quaternary charcoal records and hence fire prior to the arrival of humans (e.g. ca 130 ka: Singh et al., 1981; Dodson et al., 2005); the fact that a broadly-synchronous transition to more xerophytic vegetation has been found in New Caledonia, a region which was not settled by humans until ca 3000 years ago (Stevenson and Hope, 2005); and modeling evidence that the purported changes in vegetation cover were insufficient to cause a sustained change in Australasian climate (Pitman and Hesse, 2007). We have found no evidence of a change in fire regimes at a continental scale at the time of Aboriginal colonisation of Australia ( $50 \pm 10 \mathrm{ka})$.

Changes in Aboriginal socio-economic relationships (Lourandos, 1980, 1983) have been invoked as causing changes in fire regimes in the mid-to-late Holocene. Lourandos $(1980,1983)$ suggested that the intensification of land use by Aboriginal groups, including a shift from more nomadic to more sedentary populations, was responsible for an increase in fire from $c a .5 \mathrm{ka}$ onwards. Lynch et al. (2007) have also argued for an increase in fire associated with people, although they suggest that this occurred somewhat later after ca $3 \mathrm{ka}$. Again, we see no unequivocal relationship between inferred populations and/or the intensity of
Aboriginal occupation of the continent and fire regimes during the past $21 \mathrm{ka}$, nor do the charcoal records show a marked increase in fire during the late Holocene. The charcoal records support the idea that fire regimes were controlled by changing climate and climateinduced changes in vegetation, though it is possible that these natural changes had an impact on Aboriginal populations through control of resources.

It is possible that our failure to identify a distinctive human fingerprint on fire activity at a continental or regional scale, either at the purported time of Aboriginal settlement of the Australian continent or over the past $21 \mathrm{ka}$, reflects the use of sedimentary charcoal records or of analytical techniques that emphasize the composite signal of many individual records. It could be argued, for example, that sedimentary charcoal is an unreliable indicator of the small-scale or low intensity fires characteristic of Aboriginal burning, particularly if the material burnt was primarily nonwoody. However, studies from other areas (e.g. Brown et al., 2005) show that predominantly grassland fires are recorded by sedimentary charcoal. Furthermore, if sedimentary charcoal records fail to capture smaller-scale fires, then the use of individual charcoal records to identify human impact on the landscape is also suspect. In the absence of independent archaeological evidence, the interpretation of local charcoal signals in terms of human impact is at best equivocal as to causation even when accompanied by changes in vegetation.

It is more plausible to argue that the compositing of individual charcoal records at regional or continental scales is more likely to emphasize changes that are congruent with climate or environmental changes that operate at similar spatial scales and less likely to identify human impacts if these changes were time transgressive across a region or highly localized. The timing of Aboriginal settlement of Australia is subject to large uncertainty, and there is no clear agreement about whether settlement was time transgressive or not (see discussion in Bird et al., 2004). Furthermore, the debate about Aboriginal use of fire has focused on whether it resulted in widespread transformation of the Australian vegetation (see e.g. Flannery, 1994; Horton, 2000; Miller et al., 2005). Thus, while we cannot dismiss the idea that some individual records may contain an overprint of human influence on the local fire regime, the evidence presented here clearly demonstrates that the dominant control of fire activity is climate or climate-modulated changes in vegetation cover.

In conclusion, compilation and analysis of charcoal records from Australasia have allowed us to document changes in fire regimes over the past $70 \mathrm{ka}$ and thus to examine (and resolve) some persistent controversies about the relationship between fire, climate and humans. We cannot, as yet, explain all of the features of the fire record nor have we exhausted the potential of the current database. The ongoing improvement of the database, through e.g. extension of the geographical coverage, higher resolution sampling, and improvement of age models, will allow further questions to be addressed. Community-based regional synthesis of charcoal and vegetation records, particularly if this can be combined with carefully designed model experiments, will continue to yield new insights into the behaviour of fire in response to climate and other environmental factors on palaeo-timescales.

\section{Acknowledgements}

This paper is a contribution to the ongoing work of the QUAVIDA working group of the ARC-NZ Network for Vegetation Function, to the UK Natural Environment Research Council (NERC) funded project "Analysis of long-term Climate Change in Australia (ACACIA)" and of the Global Palaeofire Working Group, supported by NERC and the US National Science Foundation (NSF). Mike Smith 
(Australian National Museum, Canberra) and Alan Williams (ANU, Canberra) supplied the archaeological data for Fig. 7. We thank Martin Williams for his helpful comments on the manuscript.

\section{References}

Ahn, J., Brook, E.J., 2008. Atmospheric $\mathrm{CO}_{2}$ and climate on millennial time scales during the last glacial period. Science $322,83-85$.

Andrus, C.F.T., Crowe, D.E., Sandweiss, D.H., Reitz, E.J., Romanek, C.S., 2002. Otolith $\delta^{18} \mathrm{O}$ record of mid-Holocene sea surface temperatures in Peru. Science 295, 1508-1511.

Arneth, A., Harrison, S.P., Zaehle, S., Tsigaridis, K., Menon, S., Bartlein, P.J., Feichter, H., Korhola, A., Kulmala, M., O’Donnell, D., Schurgers, S., Sorvari, S., Vesala, T., 2010. Terrestrial biogeochemical feedbacks in the climate system. Nature Geoscience 3, 525-532.

Barrows, T.T., Lehman, S., Fifield, L.K., De Deckker, P., 2007. Absence of cooling in New Zealand and the adjacent ocean during the Younger Dryas Chronozone. Science 318, 86-89.

Beaufort, L., de Gardiel-Thoron, T., Linsley, B., Oppo, D., Buchet, N., 2003. Biomass burning and oceanic primary production estimates in the Sulu Sea area over the las 380,000 yr and the East Asian monsoon dynamics. Marine Geology 201, 53-65.

Bird, M.I., Hope, G., Taylor, D., 2004. Populating PEP II: the dispersal of humans and agriculture through Austral-Asia and Oceania. Quaternary International $118-119,145-163$.

Black, M., Mooney, S.D., 2006. Holocene fire history from the greater blue mountains world heritage area, New South wales, Australia: the climate, humans and fire nexus. Regional Environmental Change 6, 41-51.

Black, M.P., Mooney, S.D., Haberle, S.G., 2007. The fire, human and climate nexus in the Sydney Basin, eastern Australia. The Holocene 17, 465-478.

Black, M.P., Mooney, S.D., Attenbrow, V., 2008. Implications of a 14,200 year contiguous fire record for understanding human-climate relationships at Goochs Swamp, New South Wales, Australia. The Holocene 18, 437-447.

Bond, W.J., Keeley, J.E., 2005. Fire as a global 'herbivore': the ecology and evolution of flammable ecosystems. Trends in Ecology and Evolution 20, 387-394.

Bowman, D.M.J.S., 2000. Rainforests and flame forests: the great Australian forest dichotomy. Australian Geographical Studies 38, 327-331.

Bowman, D.M.J.S., Balch, J.K., Artaxo, P., Bond, W.J., Cochrane, M.A., D’Antonia, C.M., DeFries, R.S., Doyle, J.C., Harrison, S.P., Johnston, F.H., Keeley, J.E. Krawchuk, M.A., Kull, C.A., Marston, J.B., Moritz, M.A., Prentice, I.C., Roos, C.I., Scott, A.C., Swetnam, T.W., van der Werf, G.R., Pyne, S.J., 2009. Fire in the earth system. Science $324,481-484$

Bradstock, R.A., Williams, J.E., Gill, A.M., 2002. Flammable Australia: the fire regimes and biodiversity of a continent. Cambridge University Press, Cambridge.

Brown, K.J., Clark, J.S., Grimm, E.C., Donovan, J.J., Mueller, P.G., Hansen, B.S.C., Stefanova, I., 2005. Fire cycles in North American interior grasslands and their relation to prairie drought. PNAS $102,8865-8870$.

Bushfire Co-operative Research Centre, 2006. Climate change and its impacts on the management of bushfire. Fire Note 4, http://www.bushfirecrc.com/ publications/downloads/Firenote_climate190906.pdf.

Calvo, E., Pelejero, C., De Deckker, P., Logan, G.A., 2007. Antarctic deglacial pattern in a $30 \mathrm{kyr}$ record of sea surface temperature offshore South Australia. Geophysical Research Letters 34, 1-6.

Cary, G.J., 2002. Importance of a Changing Climate for Fire Regimes in Australia. In: Bradstock, R.A., Williams, J.E., Gill, A.M. (Eds.), Flammable Australia: the fire regimes and biodiversity of a continent. Cambridge University Press, Cambridge, pp. $26-48$

Chapman, D., 1999. Natural hazards, second ed. Oxford University Press, Oxford.

Clark, P.U., Pisias, N.G., Stocker, T.F., Weaver, A.J., 2002. The role of the thermohaline circulation in abrupt climate change. Nature 415, 863-869.

Conedera, M., Tinner, W., Neff, C., Meurer, M., Dickens, A.F., Krebs, P., 2009 Reconstructing past fire regimes: methods, applications, and relevance to fire management and conservation. Quaternary Science Reviews 28, 435-456.

Daniau, A.-L., Sánchez-Goñi, M.F., Beaufort, L., Laggoun-Défarge, F., Loutre, M.-F. Duprat, J., 2007. Dansgaard-Oeschger climatic variability revealed by fire emissions in southwestern Iberia. Quaternary Science Reviews 26, 1369-1383.

Daniau, A.-L., Sánchez-Goñi, M.F., Duprat, J., 2009. Last glacial fire regime variability in western France inferred from microcharcoal preserved in core MD04-2845, Bay of Biscay. Quaternary Research 71 (3), 385-396.

Daniau, A.-L., Harrison, S.P., Bartlein, P.J., 2010. Fire regimes during the last glacial. Quaternary Science Reviews 29, 2918-2930.

De Fries, R.S., Hansen, M.C., Townshend, J.R.G., 2000. Global continuous fields of vegetation characteristics: a linear mixture model applied to multi-year $8 \mathrm{~km}$ AVHRR data. International Journal of Remote Sensing 21 (6-7), 1389-1414.

Dodson, J.R., Robinson, M., Tardy, C., 2005. Two fine-resolution Pliocene charcoal records and their bearing on pre-human fire frequency in south-western Australia. Austral Ecology 30, 592-599.

Enright, N.J., Marsula, R., Lamont, B.B., Wissel, C., 1998. The ecological significance of canopy seed storage in fire-prone environments: a model for non-resprouting shrubs. Journal of Ecology 86, 946-959.

EPICA Community Members, 2006. One-to-one coupling of glacial climate variability in Greenland and Antarctica. Nature 444, 195-198.

Fitzsimmons, K.E., Timothy, T., Barrows, T.T., 2010. Holocene hydrologic variability in temperate southeastern Australia: an example from Lake George, New south wales. The Holocene 20, 585-597.
Flannery, T., 1994. The future eaters: an ecological history of the Australasian lands and people. Reed Books, Port Melbourne. 423pp.

Fletcher, W., Sanchez-Goñi, M.F., Allen, J.R.M., Cheddadi, R., Combourieu Nebout, N., Huntley, B., Lawson, I., Londeix, L., Magri, D., Margari, V., Müller, U.C., Naughton, F., Novenko, E., Roucoux, K., Tzedakis, P.C., 2010. Millennial-scale variability during the last glacial in vegetation records from Europe. Quaternary Science Reviews 29, 2839-2864

Gagan, M.K., Hendy, E.J., Haberle, S.G., Hantoro, W.S., 2004. Post-glacial evolution of the Indo-Pacific warm pool and El Niño-southern oscillation. Quaternary International 118-119, 127-143.

Gale, S.J., 2009. Event chronostratigraphy: a high-resolution tool for dating the recent past. Quaternary Geochronology 4, 391-399.

Gill, A.M., Groves, R.H., Noble, I.R., 1981. Fire in the Australian Biota. Australian Academy of Science, Canberra.

Haberle, S.G., 2005. A 23,000-yr pollen record from Lake Euramoo, wet tropics of NE Queensland, Australia. Quaternary Research 64, 343-356.

Harrison, S., Dodson, J.R., 1993. Climates of Australia and New Guinea since 18,000 yrs BP. In: Wright Jr., H.E., Kutzbach, J.E., Webb III, T., Ruddiman, W.F., StreetPerrott, F.A., Bartlein, P.J. (Eds.), Global climates since the last glacial maximum. University of Minnesota Press, Minnesota, pp. 265-293.

Harrison, S.P., Prentice, I.C., 2003. Climate and $\mathrm{CO}_{2}$ controls on global vegetation distribution at the last glacial maximum: analysis based on palaeovegetation data, biome modelling and palaeoclimate simulations. Global Change Biology 9, 983-1004.

Harrison, S.P., Sanchez Goni, M.F., 2010. Global patterns of vegetation response to millennial-scale variability during the last glacial: a synthesis. Quaternary Science Reviews 29, 2957-2980.

Harrison, S.P., Kutzbach, J.E., Liu, Z., Bartlein, P.J., Otto-Bleisner, B., Muhs, D., Prentice, I.C., Thompson, R.S., 2003. Mid-Holocene climates of the Americas: a dynamical response to changed seasonality. Climate Dynamics 20, 663-688.

Harrison, S.P., Marlon, J., Bartlein, P.J., 2010. Fire in the earth system. In: Dodson, J. (Ed.), Changing climates, earth systems and society. Springer-Verlag.

Hesse, P.P., Magee, J.W., van der Kaars, S., 2004. Late quaternary climates of the Australian arid zone: a review. Quaternary International 118-119, 87-102.

Hessler, I., Dupont, L., Bonnefille, R., Behling, H., González, C., Helmens, K.F., Hooghiemstra, H., Lebamba, J., Ledru, M.P., Lézine, A.M., Maley, J., Marret, F., Vincens, A., 2010. Millennial-scale changes in vegetation records from tropical Africa and South America during the last glacial. Quaternary Science Reviews 29, 2882-2899.

Horton, D.R., 2000. The Pure State of Nature: Sacred Cows, Destructive Myths and the Environment. Allen \& Unwin, Sydney, Australia.

Jackson, W.D., 1999. The Tasmanian legacy of man and fire. Papers and Proceedings of the Royal Society of Tasmania 133, 1-14.

Jones, R., 1969. Fire-stick farming. Australian Natural History 16, 224-228.

Kageyama, M., Mignot, J., Swingedouw, D., Marzin, C., Alkama, R., Marti, O., 2009 Glacial climate sensitivity to different states of the Atlantic Meridional overturning circulation: results from the IPSL model. Climate of the Past Discussions $5,1-53$.

Kageyama, M., Paul, A., Roche, D.M., Van Meerbeeck, C.J., 2010. Modelling glacial climatic millennial-scale variability related to changes in the Atlantic Meridional overturning circulation: a review. Quaternary Science Reviews 29, 2931-2956.

Kaplan, J.O., Bigelow, N.H., Bartlein, P.J., Christensen, T.R., Cramer, W., Harrison, S.P., Matveyeva, N.V., McGuire, A.D., Murray, D.F., Prentice, I.C., Razzhivin, V.Y., Smith, B. Walker, D.A., Anderson, P.M., Andreev, A.A., Brubaker, L.B. Edwards, M.E., Lozhkin, A.V., 2003. Climate change and Arctic ecosystems II: modeling, palaeodata-model comparisons, and future projections. Journal of Geophysical Research-Atmosphere 108, 8171.

Kershaw, A.P., 1986. Climatic change and aboriginal burning in north-east Australia during the last two glacial/interglacial cycles. Nature 322, 47-49.

Kershaw, A.P., Clark, J.S., Gill, A.M., D'Costa, D.M., 2002. A History of Fire in Australia. In: Bradstock, R., Williams, J., Gill, M. (Eds.), Flammable Australia; The Fire Regimes and Biodiversity of a Continent. Cambridge University Press, pp. 1-25.

Kershaw, A.P., van Der Kaars, S., Moss, P.T., 2003. Late quaternary Milankovitch-scale climatic change and variability and its impact on monsoonal Australasia. Marine Geology 201, 81-95.

Kershaw, A.P., Bretherton, S.C., van der Kaars, S., 2007. A complete pollen record of the last 230 ka from Lynch's Crater, north-east Australia. Palaeogeography, Palaeoclimatology, Palaeoecology 251, 23-45.

Kershaw, A.P., McKenzie, G.M., Porch, N., Roberts, R.G., Brown, J., Heijnis, H., Orr, L.M., Jacobsen, G., Newall, P.R., 2007. A high resolution record of vegetation and climate through the last glacial cycle from Caledonia Fen, south-eastern highlands of Australia. Journal of Quaternary Science 22, 481-500.

Koutavas, A., Lynch-Stieglitz, J., Marchitto Jr., T.M., Sachs, J.P., 2002. El Niño-like pattern in ice age tropical Pacific Sea surface temperature. Science 297, $226-230$.

Lourandos, H., 1980. Change or stability? Hydraulics, hunter gatherers and population in temperate Australia. World Archaeology 11, 245-266.

Lourandos, H., 1983. Intensification: a late Pleistocene-Holocene archaeological sequence from southwestern Victoria. Archaeology in Oceania 18, 81-94.

Lourandos, H., David, B., 1998. Comparing long-term archaeological and environmental trends: North Queensland, arid and semi-arid Australia. The Artefact 21, 105-114.

Lourandos, H., David, B., 2002. Long-term archaeological and environmental trends: a comparison from late Pleistocene-Holocene Australia. In: Kershaw, P., 
David, B., Tapper, N., Penny, D., Brown, J. (Eds.), Bridging Wallace's Line: The Environmental and Cultural History and Dynamics of the SE Asian-Australian Region. Catena Verlag, pp. 307-338.

Lynch, A.H., Beringer, J., Kershaw, A.P., Marshall, A., Mooney, S., Tapper, N., Turney, C., van der Kaars, S., 2007. Using the palaeorecord to evaluate climate and fire interactions in Australia. Annual Review of Earth and Planetary Sciences 35, 215-239.

Markgraf, V., Dodson, J.R., Kershaw, A.P., McGlone, M.S., Nicholls, N., 1992. Evolution of late Pleistocene and Holocene climates in the circum-South Pacific land areas. Climate Dynamics 6, 193-211.

Marlon, J.R., Bartlein, P.J., Carcaillet, C., Gavin, D.G., Harrison, S.P., Higuera, P.E., Joos, F., Power, M.J., Prentice, I.C., 2008. Climate and human influences on global biomass burning over the past two millennia. Nature Geoscience 1 , 697-701.

McGlone, M., Kershaw, P.A., Markgraf, V., 1992. El Nino/Southern oscillation climatic variability in Australasian and South American palaeoenvironmental records. In: Diaz, H.F., Markgraf, V. (Eds.), El Niño, Historical and Paleoclimatic Aspects of the Southern Oscillation. Cambridge University Press, Cambridge, pp. 435-562.

Miller, G.H., Fogel, M.L., Magee, J.W., Gagan, M.K., Clarke, S.J., Johnson, B.J., 2005. Ecosystem collapse in Pleistocene Australia and a human role in megafaunal extinction. Science 309, 287-290.

Millspaugh, S.H., Whitlock, C., Bartlein, P., 2004. Postglacial Fire, Vegetation, and Climate History of the Yellowstone-Lamar and Central Plateau Provinces, Yellowstone National Park. In: Wallace, L. (Ed.), After the Fires: The Ecology of Change in Yellowstone National Park. Yale University Press, pp. $10-28$.

Moy, C.M., Seltzer, G.O., Rodbell, D.T., Anderson, D.M., 2002. Variability of El Niño/ Southern oscillation activity at millennial timescales during the Holocene epoch. Nature 420, 162-165.

Muller, J., Kylander, M.E., Wust, R.A.J., Weiss, D., Martinez-Cortizas, A., LeGrande, A., Jennerjahn, T., Behling, H., Anderson, W.T., Jacobson, G., 2008. Possible evidence for wet Heinrich phases in tropical NE Australia: the Lynch's Crater deposit. Quaternary Science Reviews 27, 468-475.

New, M., Lister, D., Hulme, M., Makin, I., 2002. A high-resolution data set of surface climate over global land areas. Climate Research 21, 1-25.

Nicholson, P.H., 1981. Fire and the Australian Aborigine. In: Gill, A.M., Groves, R.H. Noble, I.R. (Eds.), Fire and the Australian Biota. Australian Academy of Science, pp. 23-54.

Pickett, E., Harrison, S.P., Hope, G., Harle, K., Dodson, J.R., Kershaw, A.P., Prentice, I.C., Backhouse, J., Colhoun, E.A., D'Costa, D., Flenley, J., Grindrod, J., Haberle, S., Hassell, C., Kenyon, C., Macphail, M., Martin, H., Martin, A.H., McKenzie, M., Newsome, J.C., Penny, D., Powell, J., Raine, I., Southern, W., Stevenson, J. Sutra, J.P., Thomas, I., van der Kaars, S., Walker, D., Ward, J., 2004. Pollen-based reconstructions of biome distributions for Australia, south east Asia and the Pacific (SEAPAC region) at 0,6000 and $18,000{ }^{14} \mathrm{C}$ yr B.P. Journal of Biogeography $31,1381-1444$.

Pitman, A.J., Hesse, P.P., 2007. The significance of large scale land cover change on the Australian palaeomonsoon. Quaternary Science Reviews 26, 189-200.

Power, M.J., Marlon, J., Ortiz, N., Bartlein, P.J., Harrison, S.P., Mayle, F.E., Ballouche, A., Bradshaw, R.H., Carcaillet, C., Cordova, C., Mooney, S., Moreno, P., Prentice, I.C., Thonicke, K., Tinner, W., Whitlock, C., Zhang, Y., Zhao, Y., Ali, A.A., Anderson, R.S., Beer, R., Behling, H., Briles, C., Brown, K.J., Brunelle, A., Bush, M., Camill, P., Chu, G.Q. Clark, J., Colombaroli, D., Connor, S., Daniau, A.-L., Daniels, M. Dodson, J., Doughty, E., Edwards, M.E., Finsinger, W., Foster, D., Frechette, J., Gaillard, M.-J., Gavin, D.G., Gobet, E., Haberle, S., Hallett, D.J., Higuera, P., Hope, G., Horn, S., Inoue, J., Kaltenrieder, P., Kennedy, L., Kong, Z.C., Larsen, C. Long, C.J., Lynch, J., Lynch, E.A., McGlone, M., Meeks, S., Mensing, S., Meyer, G. Minckley, T., Mohr, J., Nelson, D.M., New, J., Newnham, R., Noti, R., Oswald, W., Pierce, J., Richard, P.J.H., Rowe, C., Sanchez Goñi, M.F., Shuman, B.J., Takahara, H., Toney, J., Turney, C., Urrego-Sanchez, D.H., Umbanhowar, C., Vandergoes, M., Vanniere, B., Vescovi, E., Walsh, M., Wang, X., Williams, N., Wilmshurst, J., Zhang, J.H., 2008. Changes in fire regime since the Last Glacial Maximum: an assessment based on a global synthesis and analysis of charcoal data. Climate Dynamics 30, 887-907.

Power, M.J., Marlon, J.R., Bartlein, P.J., Harrison, S.P., 2010. Fire history and the Global Charcoal Database: a new tool for hypothesis testing and data exploration. Palaeogeography, Palaeoclimatology, Palaeoecology 291, 52-59.

Prentice, I.C., Harrison, S.P., 2009. Ecosystem effects of $\mathrm{CO}_{2}$ concentration: evidence from past climates. Climates of the Past 5, 297-307.

Purdie, R.W., 1977. Early stages of regeneration after burning in dry sclerophyll vegetation. I. Regeneration of the understorey by vegetative means. Australian Journal of Botany 25, 21-34.

Ramanathan, V., Carmichael, G., 2008. Global and regional climate changes due to black carbon. Nature Geoscience 1, 221-227.

Rodbell, D.T., Seltzer, G.O., Anderson, D.M., Abbott, M.B., Enfield, D.B., Newman, J.H., 1999. An $\sim 15,000$-Year record of El Niño-driven Alluviation in southwestern Ecuador. Science 283, 516-520.

Rodó, X., Rodriguez-Arias, M., 2004. El Nino-Southern oscillation: absent in the early Holocene? Journal of Climate 17, 423-426.

Russell-Smith, J., Yates, C.P., Whitehead, P.J., Smith, R., Craig, R., Allan, G.E., Thackway, R., Frakes, I., Cridland, S., Meyer, M.C.P., Gill, A.M., 2007. Bushfires 'down under': patterns and implications of contemporary Australian landscape burning. International Journal of Wildland Fire 16, 361-377.
Sanchez Goñi, M.F., Harrison, S.P., 2010. Millennial-scale climate variability and vegetation changes during the last glacial: concepts and terminology. Quaternary Science Reviews 29, 2823-2827.

Select Committee on the Recent Australian Bushfires, 2003. A Nation Charred. The Parliament of the Commonwealth of Australia, Canberra.

Shafer, S.L., Bartlein, P.J., Whitlock, C., 2005. Understanding the Spatial Heterogeneity of Global Environmental Change in Mountain Regions. In: Huber, U. Reasoner, M., Bugmann, H. (Eds.), Global Change and Mountain Regions. Springer, pp. 21-30.

Shakun, J.D., Carlson, A.E., 2010. A global perspective on Last Glacial Maximum to Holocene climate change. Quaternary Science Reviews 29, 1801-1816.

Shulmeister, J., Lees, B.G., 1995. Pollen evidence from tropical Australia for the onset of an ENSO-dominated climate at c. 4000 BP. The Holocene 5, 10-18.

Sikes, E.L., Howard, W.R., Neil, H.L., Volkman, J.K., 2002. Glacial-interglacial sea surface temperature changes across the subtropical front east of New Zealand based on alkenone unsaturation ratios and foraminiferal assemblages. Paleoceanography 17 10.1029/2001PA000640.

Singh, G., Geissler, E.A., 1985. Late Cainozoic history of vegetation, fire, lake levels and climate at Lake George, New south wales, Australia. Philosophical Transactions of the Royal Society London B 311, 379-447.

Singh, G., Kershaw, A.P., Clark, R., 1981. Quaternary Vegetation and Fire History in Australia. In: Gill, A.M., Groves, R.H., Noble, I.R. (Eds.), Fire in the Australian Biota. Australian Academy of Science, Canberra, pp. 23-54.

Smith, M.A., Williams, A.N., Turney, C.S.M., Cupper, M.L., 2008. Human-environment interactions in Australian drylands: exploratory time-series analysis of archaeological records. The Holocene 18, 389-401.

Stevenson, J., Hope, G.S., 2005. A comparison of late Quaternary forest changes in New Caledonia and northeastern Australia. Quaternary Research 64, 372-383.

Stouffer, R.J., Dixon, K.W., Spelman, M.J., Hurlin, W., Yin, J., Gregory, J.M., Weaver, A.J., Eby, M., Flato, G.M., Robitaille, D.Y., Hasumi, H., Oka, A., Hu, A., Jungclaus, J.H., Kamenkovich, I.V., Levermann, A., Nawrath, S., Montoya, M. Murakami, S., Peltier, W.R., Vettoretti, G., Sokolov, A., Weber, S.L., 2006. Investigating the causes of the response of the thermohaline circulation to past and future climate changes. Journal of Climate 19, 1365-1387.

Thevenon, F., Bard, E., Williamson, D., Beaufort, L., 2004. A biomass burning record from the West Equatorial Pacific over the last $360 \mathrm{kyr}$ : methodological, climatic and anthropic implications. Palaeogeography, Palaeoclimatology, Palaeoecology 213, 83-99.

Tinner, W., Hofstetter, S., Zeugin, F., Conedera, M., Wohlgemuth, T., Zimmermann, L. Zweifel, R., 2006. Long-distance transport of macroscopic charcoal by an intensive crown fire in the Swiss Alps - implications for fire history reconstruction. The Holocene 16, 287-292.

Tudhope, A.W., Chilcott, C.P., McCulloch, M.T., Cook, E.R., Chappell, J., Ellam, R.M. Lea, D.W., Lough, J.M., Shimmield, G.B., 2001. Variability in the El Niñosouthern oscillation through a glacial-interglacial cycle. Science 291, 1511-1517.

Turney, C.S.M., Hobbs, D., 2006. ENSO influence on Holocene aboriginal populations in Queensland, Australia. Journal of Archaeological Science 33, 1744-1748.

Turney, C.S.M., Kershaw, A.P., Moss, P., Bird, M.I., Fifield, L.K., Cresswell, R.G., Santos, G.M., di Tada, M.L., Hausladen, P.A., Zhou, Y., 2001. Redating the onset of burning at Lynch's Crater (North Queensland): implications for human settlement in Australia. Journal of Quaternary Science 16, 767-771.

Turney, C.S.M., Kershaw, P., Clemens, S., Branch, N., Moss, P., Fifield, L.K., 2004 Millennial and orbital variations of El Nino/Southern Oscillation and highlatitude climate in the last glacial period. Nature 428, 306-310.

van der Werf, G.R., Randerson, J.T., Giglio, L., Collatz, G.J., Kasibhatla, P.S Arellano Jr., A.F., 2006. Interannual variability of global biomass burning emissions from 1997 to 2004. Atmospheric Chemistry and Physics Discussions 6, 3175-3226.

van der Werf, G.R., Dempewolf, J., Trigg, S.N., Randerson, J.T., Kasibhatla, P.S Giglio, L., Murdiyarso, D., Peters, W., Morton, D.C., Collatz, G.J., Dolman, A.J., De Fries, R.S., 2008a. Climate regulation of fire emissions and deforestation in equatorial Asia. Proceedings of the National Academy of Sciences of the United States of America 105, 20350-20355.

van der Werf, G.R., Randerson, J.T., Giglio, L., Gobron, N., Dolman, A.J., 2008b Climate controls on the variability of fires in the tropics and subtropics. Global Biogeochemical Cycles 22. doi:10.1029/2007GB003122.

Vandergoes, M.J., Newnham, R.M., Preusser, F., Hendy, C.H., Lowell, T.V. Fitzsimons, S.J., Hogg, A.G., Kasper, H.U., Schluechter, C., 2005. Regional insolation forcing of Late Quaternary climate change in the Southern Hemisphere. Nature 436, 242-245.

Webb III, T., Anderson, K.H., Bartlein, P.J., Webb, R.S., 1998. Late quaternary climate change in eastern North America: a comparison of pollen-derived estimates with climate model results. Quaternary Science Reviews 17, 587-606.

Williams, N.J., 2005. The environmental reconstruction of the last glacial cycle at Redhead Lagoon in coastal eastern Australia. PhD thesis, University of New South Wales, Sydney, Australia.

Williams, A.A.J., Karoly, D.J., Tapper, N., 2001. The sensitivity of Australian fire danger to climate change. Climatic Change 49, 171-191.

Williams, M., Cook, E., van der Kaars, S., Barrows, T., Shulmeister, J., Kershaw, P., 2009. Glacial and deglacial climatic patterns in Australia and surrounding regions from 35,000 to 10,000 years ago reconstructed from terrestrial and near-shore proxy data. Quaternary Science Reviews 28, 2398-2419. 


\section{Database References}

Aitken, D.L., Kershaw, A.P., 1993. Holocene vegetation and environmental history of Cranbourne Botanic Garden, Victoria. Proceedings of the Royal Society of Victoria 105 (1), 67-80.

Anker, S.A., Colhoun, E.A., Barton, C.E., Petersen, M., Barbetti, M., 2001. Holocene vegetation and paleoclimatic and Paleomagnetic history from Lake Johnsten, Tasmania. Quaternary Research 56 (2), 264-274.

Anshari, G., Kershaw, A.P., Van der Kaars, S., 2001. A late Pleistocene and Holocene pollen and charcoal record from peat swamp forest, Lake Sentarum Wildlife Reserve, west Kalimantan, Indonesia. Palaeogeography, Palaeoclimatology, Palaeoecology 171, 637-655.

Athens, J.S., Ward, J.V., 2005. Palau Compact Road archaeological investigations, Babeldaob island, Republic of Palau. Unpublished report.

Black, M.P., 2001. Fire, vegetation, humans and climate: a record of change during the past millennium from the Kings Tablelands. Unpublished BSc Hons thesis School of Geography, UNSW, Sydney

Black, M.P., Mooney, S.D., 2006. Holocene fire history from the Greater Blue Mountains world Heritage Area, NSW, Australia: the climate, humans and fire nexus. Regional Enviroment Change 6, 41-51.

Black, M.P., Mooney, S.D., Martin, H.A., 2006. A >43,000-year vegetation and fire history from Lake Baraba, New south wales, Australia. Quaternary Science Reviews 25, 3003-3016.

Boyd, W.E., 1990. Quaternary pollen analysis in the arid zone of Australia: Dalhousie Springs, central Australia. Review of Palaeobotany and Palynology 64 (1-4) 331-341.

Buckman, S., Brownlie, K.C., Bourman, R.P., Murray-Wallace, C.V., Morris, R.H. Lachlan, T.J., Roberts, R.G., Arnold, L.J., Cann, J.H., 2009. Holocene palaeofire records in a high-level, proximal valley-fill (Wilson Bog), Mount Lofty Ranges, South Australia. The Holocene 19, 1017-1029.

Builth, H., Kershaw, A.P., White, C., Roach, A., Hartney, L., McKenzie, M., Lewis, T., Jacobsen, G., 2008. Environmental and cultural change on the Mt Eccles lavaflow landscapes of southwest Victoria, Australia. The Holocene 18, 413-424.

Chalson, J.M., 1991. The late Quaternary vegetation and climatic history of the blue Mountains, N.S.W. Australia. Unpublished PhD thesis, University of N.S.W.

Clark, D.J., 1997. A palaeoenvironmental study of Thirlmere Lakes: applications to contemporary vegetation management. Unpublished BSc Hons Thesis, School of Geography, UNSW, Sydney

Clark, R.L., 1983. Pollen and charcoal evidence for the effects of Aboriginal burning on the vegetation of Australia. Archaeology in Oceania 18 (2), 32-37.

Clark, G., Hope, G.S., 1997. Preliminary report on archaeological and palaeoenvironmental investigations in northern Lau (Mago, Yacata-Kaibu and Vatuvara), Fiji. Unpublished Report to National Museum, Fiji.

Colhoun, E.A., 1985. Pre-last glaciation maximum vegetation history at Henty Bridge, western Tasmania. New Phytologist 100, 681-690.

Colhoun, E.A., 1992. Late glacial and Holocene vegetation history at Poets Hill Lake, western Tasmania. Australian Geographer 23, 11-23.

Colhoun, E.A., Pola, J.S., Barton, C.E., Heijnis, H., 1999. Late-Pleistocene vegetation and climate history of Lake Selina, western Tasmania. Quaternary International 57 (8), 5-23.

Colhoun, E.A., Van de Geer, G., Hannan, D., 1991. Late glacial and Holocene vegetation history at Dublin bog north-central Tasmania. Australian Geographica Studies 29 (2), 337-354.

Cupper, M.L., 2005. Last glacial to Holocene evolution of semi-arid rangelands in southeastern Australia. The Holocene 15, 541-553.

Cupper, M.L., 2006. Luminescence and radiocarbon chronologies of playa sedimentation in the Murray Basin, southeastern Australia. Quaternary Science Reviews 25, 2594-2607.

D'Costa, D.M., 1997. The reconstruction of Quaternary vegetation and climate on king island, Bass Strait, Australia. Unpublished PhD Thesis, Monash University.

D’Costa, D.M., Edney, P., Kershaw, P., De Deckker, P., 1989. Late Quaternary palaeoecology of Tower Hill, Victoria, Australia. Journal of Biogeography 16, 461-482.

D'Costa, D.M., Grindrod, J., Ogden, R., 1993. Preliminary environmental reconstructions from the late Quaternary pollen and mollusc assemblages at Egg Lagoon, king island, Bass Strait. Australian Journal of Ecology 18, 351-366.

de Montford, M., 2008. Late Holocene fire history: Human impacts and climate variability at Wingecarribee Swamp. Unpublished BSc Hons thesis, School of Biological, Earth \& Environmental Sciences, UNSW, Sydney.

Devoy, R.J., Dodson, J.R., Thom, B.G., Nichol, S., 1994. Holocene environments in the Hawkesbury Valley, New South Wales: a comparison of terrestrial and marine records. Quaternary Science Reviews 13, 241-256.

Dickinson, W.R., Burley, D.V., Nunn, P.D., Anderson, A., Hope, G., De Biran, A., Burke, C., Matararaba, S., 1998. Geomorphic and archaeological landscapes of the Sigatoka dunes site, Viti Levu, Fiji: interdisciplinary investigations. Asian Perspectives 31, 1-31.

Dodson, J.R., 1986. Holocene vegetation and environments near Goulburn, New south wales. Australian Journal of Botany 34, 231-249.

Dodson, J.R., 2001. A vegetation and fire history in a subalpine woodland and rainforest region, Solomons Jewel Lake, Tasmania. The Holocene 11, 111-116.

Dodson, J.R., Lu, J.J., 2000. A late Holocene vegetation and environment record from Byenup Lagoon, south-western Australia. Australian Geographer 31 (1) 41-54.

Dodson, J.R., De Salis, I., Myers, C.A., Sharp, A.J., 1994a. A thousand years of environmental change and human impact in the alpine zone at Mount Kosciusko New South Wales. Australian Geographer 25, 77-87.
Dodson, J.R., Frank, K., Fromme, M., Hickson, D., McRae, V., Mooney, S., Smith, J.D., 1994b. Environmental Systems and human impact at Cobrico Crater, southwestern Victoria. Australian Geographical Studies 32, 27-40.

Dodson, J.R., Greenwood, P.G., Jones, R.L., 1986. Holocene forest and wetland dynamics at Barrington Tops, NSW. Journal of Biogeography 13, 538-563.

Dodson, J.R., McRae, V.M., Molloy, K., Roberts, F., Smith, J.D., 1993. Late Holocene human impact on two coastal environments in NSW, Australia: a comparison of Aboriginal and European impacts. Vegetation History and Archaeobotany 2, 89-100.

Dodson, J.R., Mitchell, F.J.G., Bogeholz, H., Julian, N., 1998. Dynamics of temperate rainforest from fine resolution pollen analysis, upper Ringarooma river, northeastern Tasmania. Australian Journal of Ecology 23 (6), 550-561.

Dodson, J.R., Roberts, F.K., De Salis, T., 1994c. Palaeoenvironment and human impact at Burraga swamp in montane rainforest, Barrington tops national park, New south wales. Australia Australian Geographer 25, 161-169.

Donders, T.H., Wagner, F., Dilcher, D.L., Visscher, H., 2006. Mid- to late-Holocene El Nino-Southern Oscillation dynamics reflected in the subtropical terrestrial realm. Proceedings of the National Academy of Sciences 102 (31), 10904-10908.

Dyson, W.D., 1995. A pollen and vegetation history from Lake Dove, western Tasmania. Unpublished Hons thesis, geography, UNSW, Kensington.

Edney, P.A., Kershaw, A.P., De Deckker, P., 1990. A late Pleistocene and Holocene vegetation and environmental record from Lake Wangoom, western plains of Victoria, Australia. Palaeogeography, Palaeoclimatology, Palaeoecology 80, $325-343$.

Ellison, J., 2005. Holocene palynology and sea-level change in two estuaries in Southern Irian Jaya. Palaeogeography, Palaeoclimatology, Palaeoecology 220, 291-309.

Field, J.H., Dodson, J.R., Prosser, I.P., 2002. A late Pleistocene vegetation history from the Australian semi-arid zone. Quaternary Science Reviews 21 (8), 1023-1037.

Gell, P.A., Stuart, I.M., Smith, J.D., 1993. The response of vegetation to changing fire regimes and human activity in the Delegate River catchment, East Gippsland, Victoria. The Holocene 3, 150-160.

Genever, M., Grinrod, J., Barker, B., 2003. Holocene palynology of Whitehaven swamp, Whitsunday island, Queensland, and implications for the regional archaeological record. Palaeogeography, Palaeoclimatology. Palaeoecology 201, $141-156$.

Gillespie, R., Magee, J.W., Luly, J.G., Dlugokencky, E., Sparks, R.J., Wallace, G., 1991 AMS radiocarbon dating in the study of arid environments: examples from Lake Eyre, south Australia. Palaeogeography, Palaeoclimatology, Palaeoecology 84, 333-338.

Haberle, S., 2005. A 23,000-yr pollen record from Lake Euramoo, wet tropics of NE Queensland, Australia. Quaternary Research 64, 343-356.

Haberle, S., Hope, G.S., DeFretes, Y., 1991. Environmental change in the Baliem valley, Irian Jaya, Indonesia. Journal of Biogeography 18, 25-40.

Haberle, S.G., 1996. Explanations for palaeoecological changes on the northern plains of Guadalcanal, Solomon Islands: the last 3200 years. The Holocene 6, 333-338.

Haberle, S.G., 2007. Was the early Holocene characterised by "El Niño-like" or "La Niña-like" conditions? Evidence from terrestrial archives in the western Pacific Warm Pool region. Quaternary International 167-168 (Supplement (XVII INQUA Congress)) 150.

Haberle, S.G., Ledru, M.P., 2001. Correlations among charcoal records of fires from the past 16,000 Years in Indonesia, Papua New Guinea, and central and south America. Quaternary Research 55 (1), 97-104.

Haberle, S.G., Hope, G.S., Van der Kaars, W.A., 2001. Biomass burning in Indonesia and Papua New Guinea: natural and human induced fire events in the fossil record. Palaeogeography, Palaeoclimatology, Palaeoecology 171, 259-268.

Haberle, S.G., Stevenson, J., Black, M.P., Mooney, S.D., Sniderman, J.M.K., Willis, K.J., Bennett, K.D., 2005. Fire in the tropics: a paleoclimatic assessment of charcoal records from the western Pacific Warm Pool region. In Prog. Abstr. Of the AGU Chapman.

Hajdas, I., Lowe, D.J., Newnham, R.M., Bonani, G., 2006. Timing of the late-glacial climate reversal in the Southern Hemisphere: a high-precision radiocarbon chronology for Kaipo bog, New Zealand. Quaternary Research 65, 340-345.

Harle, K.J. Heijnis, H. Chisari, R. Kershaw, A.P. Zoppi, U., Jacobsen, G. 2002. A chronology for the long pollen record from Lake Wangoom, western Victoria (Australia) as derived from uranium/thorium disequilibrium dating. Journal of Quaternary Science 17, 707-720.

Head, L. 1988. Holocene Vegetation, fire and environmental history of the Discovery Bay region, southwestern Victoria. Australian Journal of Ecology 13, 21-49.

Head, L., Stuart, I.M.F., 1980. Change in the Aire-palaeoecology and Prehistory in the Aire Basin, southwestern, Victoria. Monash Publications in Geography 24,1-97.

Hooley, A.D., Southern, W., Kershaw, A.P., 1980. Holocene vegetation and environments of Sperm Whale head, Victoria, Australia. Journal of Biogeography 7, 349-362.

Hope, G., 2005a. Rock Arch and Gallaher's swamps: core samples collected in the Avon/Robertson catchments 17 March 2005. Unpublished report to Sydney Water.

Hope, G., 2009. Environmental change and fire in the Owen Stanley ranges, Papua New Guinea. Quaternary Science Reviews 28, 2261-2276.

Hope, G., Macphail, M., Keaney, B., 2005. Snowy Flat and Ginini Bogs, Brindabella Range, Australian Capital Territory. Unpublished report to environment ACT and Ecowise Services. 
Hope, G., Pask, J., 1998. Tropical vegetational change in the late Pleistocene of New Caledonia. Palaeogeography, Palaeoclimatology, Palaeoecology 142,1-21.

Hope, G., Stevenson, J., Southern, W., 2009. Vegetation histories from the Fijian islands: alternative records of human impact. Terra Australis 31, 63-87.

Hope, G.S., 1996a. Quaternary change and historical biogeography of Pacific Islands. In: KeastA.Miller, S.E. (Ed.), The Origin and Evolution of Pacific Island Biotas, New Guinea to Eastern Polynesia: Patterns and Process. SPB Publ, Amsterdam, pp. 165-190.

Hope, G.S., 1996b. A reconnaissance survey of potential sites for pollen analysis on Aneityum Island, southern Vanuatu. Unpublished Report to the Vanuatu Cultural Centre, Vanuatu.

Hope, G.S., 1998. Early fire and forest change in the Baliem valley, Irian Jaya, Indonesia. Journal of Biogeography 25 (3), 453-461.

Hope, G.S., 1999. Vegetation and fire response to late Holocene human occupation in island and mainland north west Tasmania. Quaternary International 59, 47-60.

Hope, G.S., 2001. Environmental change in the late Pleistocene and later Holocene at Wanda site, Soroako, south Sulawesi, Indonesia. Palaeogeography, Palaeoclimatology, Palaeoecology 171 (3-4), 129-145.

Hope, G.S., 2005b. Tom Gregory bog, an infilled meander of the upper Cotter River, Australian Capital Territory. Unpublished report to ECOWISE ACT and environment ACT.

Hope, G.S., 2007a. The History of Human Impact on New Guinea. In: Marshall, A.J., Beehler, B.M. (Eds.). The Ecology of Papua, Singapore, pp. 1087-1097.

Hope, G.S., 2007b. Palaeoecology and Palaeoenvironments of Papua. In: Marshall, A.J., Beehler, B.M. (Eds.), The Ecology of Papua. Periplus Editions, Singapore.

Hope, G.S., Aplin, K., 2005. Environmental Change in the Aru Islands. In: O'Connor, S., Spriggs, M., Veth, P. (Eds.), The Archaeology of the Aru Islands Eastern Indonesia. Pandanus Press, Canberra, pp. 25-40.

Hope, G.S., Tulip, J., 1994. A long vegetation history from lowland Irian Jaya, Indonesia. Palaeogeography, Palaeoclimatology, Palaeoecology 109, 385-398.

Hope, G.S., Clark, R.L., 2008. A Tale of Two Swamps: Subalpine Peatlands in the Kelly-Scabby Area of Namadgi National Park. In: McCueK.Lenz, S. (Ed.), Corridors for Survival in a Changing World. National Parks Assoc. ACT, Canberra, pp. 61-76.

Hope, G.S., Coddington, J., O’Dea, D., 2006a. Estuarine development and human occupation at Bobundara Swamp, Tilba Tilba, New South Wales, Australia. In: Lillie, M., Ellis, S. (Eds.), Wetland Archaeology and Environments: Regional Issues, Global Perspectives. Oxbow, Oxford, pp. 258-274.

Hope, G.S., O'Dea, D., Southern, W., 1996. Le pacifique de 5000 à 2000 avant le présent. Suppléments à l'histoire d'une colonisation. The Pacific from 5000 to 2000 BP. Colonisation and transformations. Actes du colloque Vanuatu. Editions de l'ORSTOM, Paris.

Hope, G.S., O’Dea, D., Southern, W., 1999. Holocene Vegetation Histories in the Western Pacific - Alternative Records of Human Impact. In: Lilley, I., Galipaud, J.-C. (Eds.), Colonisation of the Pacific 3000-2000 BP. ORSTOM, Port Vila.

Hope, G.S., Stevenson, J., Haberle, S., 2006b. Palaeoecology of Blundells flat, ACT. Unpublished Report to Ecowise Services and ACT Forests.

Horn, D., 2005. The fire history of Broughton Island. Unpublished BSc Hons AHESIS, School of Biological, Earth \& Environmental Sciences, UNSW, Sydney.

Jenkins, M.A., 1992. A Late Holocene Vegetation Record from an Interdunal Swamp, Mornington Peninsula, Victoria. Unpublished M. Env.Sci. Thesis, Department of Geography and Environmental Science, Monash University, Melbourne.

Jones, R.L., 1990. Late Holocene vegetational changes on the Illawarra coastal Plain, New south wales, Australia. Review of Palaeobotany and Palynology 65, 37-46.

Kemp, J., 1993. The end of the Pleistocene in the southern tablelands, southeastern Australia. Unpublished BA thesis, Dept geography, ANU, Australia,

Kenyon, C.E., 1989. A Late Pleistocene and Holocene Palaeoecological Record from Boulder Flat, East Gippsland. BSc Honours thesis, Department of Botany and Zoology, Monash University, Melbourne.

Kershaw, A.P., Bretherton, S.C., van der Kaars, S., 2007a. A complete pollen record of the last 230 ka from Lynch's Crater, north-east Australia. Palaeogeography, Palaeoclimatology, Palaeoecology 251, 23-45.

Kershaw, A.P., McKenzie, G.M., Porch, N., Roberts, R.G., Brown, J., Heijnis, H., Orr, M.L., Jacobsen, G., Newall, P.R., 2007b. A high-resolution record of vegetation and climate through the last glacial cycle from Caledonia Fen, southeastern highlands of Australia. Journal of Quaternary Science 22, 481-500.

Kodela, P.G., 1996. The vegetation of the Robertson Plateau, New South Wales: historical and contemporary issues. Unpublished PhD thesis, UNSW, Australia, Sydney.

Kodela, P.G., Dodson, J.R., 1988. A late Holocene vegetation and fire record from Kuring-gai Chase national park, NSW. Proceedings of the Linnean Society 110, 317-326.

Ladd, P.G., 1978. Vegetation history at Lake Curlip in lowland eastern Victoria, from 5200 B.P. To present. Australian Journal of Botany 26, 393-414.

Ladd, P.G., Orchiston, D.W., Joyce, E.B., 1992. Holocene vegetation historyof Flinders Island. New Phytologist 122, 757-767.

Latham, M., Hughes, P.J., Hope, G., Brookfield, M., 1983. Sedimentation in the swamps of Lakeba and its implications for erosion and human occupation of the island. In: Latham, M., Brookfield, H.C. (Eds.), The Eastern Islands of Fiji-a study of the natural environment, its use and man's influence on its evolution. Travaux et Documents de l'ORSTOM, Paris, pp. 103-120.

Leahy, P.J., Tibby, J., Kershaw, A.P., Heijnis, H., Kershaw, J.S., 2005. The impact of European settlementt on Bolin Billabong, a Yarra River floodplain lake, Melbourne, Australia. River Research Applications 21, 131-149.
Longmore, M.E., 1997. Quaternary palynological records from Perched Lake sediments, Fraser island, Queensland, Australia: rainforest, forest history and climatic control. Australian Journal of Botany 45 (3), 507-526.

Longmore, M.E., Torgersen, T., O'Leary, B.M., Luly, J.G., 1986. Caesium-137 mobility in the sediments of the playa Lake Tyrrell, northwestern Victoria 1:Stratigraphy and caesium-137 mobility in the upper sediments. Palaeogeography, Palaeoclimatology, Palaeoecology 54, 181-196.

Luly, J.G., 1993. Holocene palaeoclimates and environment around Lake Tyrrell, semi-arid northwestern Victoria, Australia. Journal of Biogeography 20, 587-598.

Luly, J.G., 1998. Modern pollen dynamics and surface processes in salt lake environments of semi-arid northwestern Victoria, Australia. Review of Palaeobotany and Palynology 97, 301-318.

Luly, J.G., 2001. On the equivocal fate of late Pleistocene Callitris Vent. (Cupressaceae) woodlands in arid south Australia. Quaternary International 83-85 $155-168$.

Luly, J.G., Jacobsen, G.C., 2000. Two new AMS dates from Lake Frome, arid south Australia. Quaternary Australasia 18, 29-33.

Luly, J.G., Bowler, J.M., Head, M.J., 1986. A radiocarbon chronology from the playa Lake Tyrrell, northwestern Victoria, Australia. Palaeogeography, Palaeoclimatology, Palaeoecology 54, 171-180.

Martin, A.R.H., 1994. Kurnell Fen: an eastern Australian coastal wetland, its Holocene vegetation, relevant to sea-level change and aboriginal land use. Review of Palaeobotany and Palynology 80, 311-332.

Mason, S., 2004. Late Holocene fire activity at Mellong swamp, Wollemi national park, NSW. Unpublished BSc Hons thesis, School of Biological, Earth \& Environmental Sciences, UNSW, Sydney.

McGlone, M.S., 2001. The origin of the indigenous grasslands of southeastern South Island in relation to pre-human woody ecosystems. New Zealand Journal of Ecology 25, 1-15.

McGlone, M.S., 2009. Postglacial history of New Zealand wetlands and implications for their conservation. New Zealand Journal of Ecology 33, 1-23.

McGlone, M., Wilmshurst, J.M., 1999. A Holocene record of climate, vegetation change and peat bog development, east Otago, South Island, New Zealand. Journal of Quaternary Science 14 (3), 239-254.

McGlone, M.S., Turney, C.S.M., Wilmshurst, J.M., 2004. Late glacial and Holocene vegetation and climatic history of the Cass Basin, central south island, New Zealand. Quaternary Research 62, 267-279.

McKenzie, G.M., 1997. The late Quaternary vegetation history of the south-central highlands of Victoria, Australia:1. Sites above 900m. Australian Journal of Ecology 22, 19-36.

McKenzie, G.M., 2002. The late Quaternary vegetation history of the south-central highlands of Victoria, Australia. II. Sites below $900 \mathrm{~m}$. Austral Ecology 27, $32-54$.

McKenzie, G.M., Kershaw, A.P., 1997. A vegetation history and quantitative estimate of Holocene climate from Chapple Vale, in the Otway region of Victoria. Australian Journal of Botany 45, 565-581.

McKenzie, G.M., Kershaw, A.P., 2000. The last glacial cycle from Wyelangta, the Otway region of Victoria, Australia. Palaeogeography, Palaeoclimatology, Palaeoecology 155, 177-193.

McKenzie, G.M., Kershaw, A.P., 2004. A Holocene pollen record from cool temperate rainforest, Aire crossing, the Otway region of Victoria, Australia. Review of Palaeobotany and Palynology 132, 281-290.

Mooney, S.D., Maltby, E.L., 2006. A synthesis of two proxy records revealing the late Holocene fire history at a site on the central coast of New South Wales, Australia. Austral Ecology 31, 682-695.

Mooney, S.D., Radford, K.L., Hancock, G., 2001. Clues to the 'burning question': preEuropean fire in the Sydney coastal region from sedimentary charcoal and palynology. Ecological Management and Restoration 2 (3), 203-212.

Mooney, S.D., Watson, J.R., Dodson, J.R., 1997. Late Holocene environmental change in an upper montane area of the Snowy Mountains, New south wales. Australian Geographer 28, 185-200.

Mooney, S.D., Webb, M., Attenbrow, V., 2007. A comparison of charcoal and archaeological information to address the influences on Holocene fire activity in the Sydney Basin. Australian Geographer 38, 177-194.

Moss, P.T., Kershaw, A.P., 2000. The last glacial cycle from the humid tropics of northeastern Australia: comparison of a terrestrial and marine record. Palaeogeography, Palaeoclimatology, Palaeoecology 155, 155-176.

Moss, P.T., Ian, A., Thomas, B., Macphail, M., 2007. Late Holocene vegetation and environments of the Mersey valley, Tasmania. Australian Journal of Botany 55, 74-82.

Newnham, R.M., Lowe, D.J., 2000. A fine-resolution pollen record of late glacial climate reversal from New Zealand. Geology 28, 759-762.

Newnham, R.M., Lowe, D.J., Giles, T.M., Alloway, B.V., 2007a. Vegetation and climate of Auckland, New Zealand, since ca 32,000 cal yr ago: support for an extended LGM. Journal of Quaternary Science 22, 517-534.

Newnham, R.M., Lowe, D.J., Matthews, B.W., 1998. A late Holocene and prehistoric tephropalynological record of environmental change from Lake Waikaremoana, New Zealand. The Holocene 8, 443-454.

Newnham, R.M., Vandergoes, M.J., Hendy, C.H., Lowe, D.J., Preusser, F., 2007b. A terrestrial palynological record for the last two glacial cycles from southwestern New Zealand. Quaternary Science Reviews 26, 517-535.

Ogden, J., Baher, L., McGlone, M., 1998. Fire, forest regeneration and links with Early Human habitation: evidence from New Zealand. Annals of Botany 81, 687-696.

Penny, D., Kealhofer, L., 2004. Microfossil evidence of land-use intensification in north Thailand. Journal of Archaeological Science 32, 169-182. 
Petherick, L., McGowan, H., Moss, P., 2008. Climate variability during the Last Glacia Maximum in eastern Australia: evidence of two stadials? Journal of Quaternary Science 23, 787-802.

Pittock, J., 1989. Palaeoenvironments of the Mt. Disappointment Plateau (Kinglake west, Victoria), from the late Pleistocene. Unpublished BSc (Hons.) thesis, Dept. Of geography and environmental Science, Monash University, Melbourne.

Polach, H., Singh, G., 1980. Contemporary 14C levels and their significance to sedimentary history of Bega Swamp, New South Wales. Radiocarbon 22, 398-409.

Radclyffe, E., 1993. Fire and people in Jervis Bay: toward a fire history in an occupied landscape. Unpublished BA Honours thesis, Department of Geography, Australian National University.

Raine, J.I., 1974. Pollen sedimentation in relation to Quaternary vegetation history of the Snowy Mountains of New south wales. Unpublished PhD Thesis, Australian National University, Canberra.

Reid, M.A., Sayer, C.D., Kershaw, A.P., Heiinis, H., 2007. Palaeolimnological evidence for submerged plant loss in a floodplain lake associated with accelerated catchment soil erosion (Murray River, Australia). Journal of Paleolimnology 38, $191-208$.

Robbie, A., 1998. The history of the vegetation from the palynology of Mountain Lagoon, blue Mountains, New south wales. Unpubl. BSc thesis, Biological Science, UNSW, Sydney

Robertson, M., 1986. Fire regimes and vegetation dynamics: a case study examining the potential of fine resolution palaeoecological techniques. Unpublished BA (Hons) Thesis, Dept. of Geography, Monash University, Melbourne.

Rogers, J., Hope, G.S., 2006. The Holocene infill of Nursery swamp, a large sedgeland in the Australian Capital Territory. Unpublished Report to Environment ACT.

Rowe, C., 2006a. A Holocene history of vegetation change in the western Torres Strait region, Queensland, Australia. Unpublished PhD Thesis, School of Geography and Environmental Science, Monash University.

Rowe, C. 2006b. Landscapes in western Torres Strait history. In: David, B., Barker, B. McNiven, I. (Eds.), The Social Archaeology of Indigenous Societies. Aboriginal Studies Press, Canberra, pp. 270-287.

Rowe, C., 2007. A palynological investigation of Holocene vegetation change in Torres Strait, seasonal tropics of northern Australia. Palaeogeography, Palaeoclimatology, Palaeoecology 25 (1), 83-103.

Singh, G., Geissler, E.A., 1985. Late Cainozoic history of vegetation, fire, lake levels and climate at Lake George, New South ales, Australia. Philosophical Transactions Royal Society London B 311, 379-447.

Singh, G., Kershaw, A.P., Clark, R., 1981. Quaternary vegetation and fire history in Australia. In: Gill, A.M., Groves, R.A., Noble, I.R. (Eds.), Fire and the Australian Biota. Australian Academy of Science, Canberra, pp. 23-54.

Singh, G., Luly, J.G., 1991. Changes in vegetation and seasonal climate since the last full glacial at Lake Frome, South Australia. Palaeogeography, Palaeoclimatology, Palaeoecology 84, 75-86.
Smeulders, D.M., 1999. A late Holocene environmental reconstruction using lake sediments from Hopwood's Lagoon, NSW. Unpublished BSc Hons thesis, School of Geography, UNSW, Sydney.

Stevenson, J., 1998. Late quaternary environmental change and the impact of Melanesian colonisation in New Caledonia. Unpublished PhD thesis, Geography, University of New South Wales.

Stevenson, J., 2004. A late Holocene record of human impact from the southwest coast of New Caledonia. The Holocene 14, 888-898.

Stevenson, J., Hope, G.S., 2005. A comparison of late Quaternary forest changes in New Caledonia and northeastern Australia. Quaternary Research 64, 372-383.

Stevenson, J., Siringan, F., Finn, J., Madulid, M., Heijnis, H., 2009. Paoay Lake, northern Luzon, the Philippines: a record of Holocene environmental change. Global Change Biology. doi:10.1111/j.1365-2486.2009.02039.x.

Thevenon, F., Bard, E., Williamson, D., Beaufort, L., 2004. A biomass burning record from the West Equatorial Pacific over the last $360 \mathrm{kyr}$ : methodological, climatic and anthropic implications. Palaeogeography, Palaeoclimatology, Palaeoecology 213, 83-99.

Thomas, I., Hope, G.S., 1994. An example of Holocene vegetation stability from Camerons Lagoon, a near treeline site on the Central Plateau, Tasmania. Australian Journal of Ecology 19, 150-158.

Van de Geer, G., Fitzsimons, S.J., Colhoun, E.A., 1989. Holocene to middle last glaciation vegetation history at Newell Creek, Western Tasmania. New Phytologist 111, 549-558.

van der Kaars, S., De Deckker, P., 2002. A late quaternary pollen record from deep sea core Fr10/95, GC17 offshore Cape Range Peninsula, northwestern Western Australia. Review of Palaeobotany and Palynology 120, 17-39.

van der Kaars, S., Wang, X., Kershaw, P., Guichard, F., Setiabudi, D.A., 2000. A late quaternary palaeoecological record from the Banda Sea, Indonesia: patterns of vegetation, climate and biomass burning in Indonesia and northern Australia. Palaeogeography, Palaeoclimatology, Palaeoecology 155, 135-153.

Vandergoes, M.J., Newnham, R.M., Preusser, F., Hendy, C.H., Lowell, T.V., Fitzsimons, S.J., Hogg, A.G., Kasper, H.U., Schlüchter, C., 2005. Regional insolation forcing of late quaternary climate change in the southern hemisphere. Nature 436, 242-245.

Wang, X., van der Kaars, S., Kershaw, P., Bird, M., Jansen, F., 1999. A record of fire, vegetation and climate through the last three glacial cycles from Lombok Ridge core G6-4, eastern Indian Ocean, Indonesia. Palaeogeography, Palaeoclimatology, Palaeoecology 147, 241-256.

Williams, N.J., 2005. The environmental reconstruction of the last glacial cycle at Redhead Lagoon in coastal, eastern Australia. Unpublished PhD thesis, University of Sydney, Australia.

Worthy, M., Macphail, M., Wasson, R., Hope, G., Olley, J., 2005. Environmental change of the alluvial infill of Muellers Rock Creek, upper Cotter valley, ACT. Unpublished report to ECOWISE ACT and environment ACT. 